\title{
Noncoding RNAs in neurodegeneration
}

Evgenia Salta ${ }^{1,2}$ and Bart De Strooper ${ }^{1,2,3}$

${ }^{1}$ VIB Center for Brain and Disease Research, Herestraat 49, 3000, Leuven, Belgium

${ }^{2} \mathrm{KU}$ Leuven, Department of Neurosciences, Leuven Institute for Neuroscience and Disease (LIND), Herestraat 49, 3000, Leuven, Belgium

${ }^{3}$ Dementia Research Institute, University College London, Gower Street, WC1E 6BT, London, United Kingdom

Evgenia.Salta@cme.vib-kuleuven.be; Bart.DeStrooper@cme.vib-kuleuven.be 
Abstract | The emerging complexity of the transcriptional landscape poses great challenges to our conventional preconceptions of how the genome regulates brain function and dysfunction. Non-protein-coding RNAs (ncRNAs) confer a high level of intricate and dynamic regulation of various molecular processes in the CNS, ranging from neurodevelopment to brain ageing, from synapse function to cognitive performance, and from health to disease. ncRNA-mediated processes may be involved in various aspects of the pathogenesis of neurodegenerative disorders. Understanding these events may help to develop novel diagnostic and therapeutic tools. Here, we provide an overview of the complex mechanisms that are affected by diverse ncRNA classes that have been implicated in neurodegeneration. 
Novel high-resolution and high-throughput technologies, such as tiling arrays and deeptranscriptome sequencing, have revealed that complex genomes give rise to noncoding RNAs (ncRNAs) ${ }^{1,2}$ (FIG. 1). ncRNAs are particularly abundant in the $\mathrm{CNS}^{3-5}$. It has been estimated that $40 \%$ of long ncRNA (IncRNAs) genes are specifically expressed in brain tissue, and other types of ncRNAs, such as circular RNAs (circRNAs) and certain microRNAs (miRNAs), have also been reported to be enriched in the CNS (and some specifically at synapses) ${ }^{6-10}$. Remarkably, research in the field of neurodegeneration has until now largely focused on a small percentage of the approximately 20,000 protein-coding genes. Proteincoding genes occupy less than $2 \%$ of the whole genome ${ }^{11}$, which inevitably raises the question as to whether we are overlooking an important part of the biology that underlies neurodegenerative disorders.

The functional interrogation of the rapidly increasing number of annotated ncDNA sequences is a formidable challenge and therefore critical experimental validation of the emerging evidence is required (BOX 1). Nevertheless, recent years have seen a surge of studies underscoring the vital roles of ncRNAs in brain evolution, development, homeostasis, stress responses and plasticity ${ }^{12-23}$. Indeed, loss-of-function studies have provided examples of the importance of ncRNA-mediated regulation in brain function ${ }^{24-28}$. Moreover, ncRNAs in adult mouse brain often exhibit region- and stage-specific expression patterns ${ }^{29,30}$, are dynamically regulated by neuronal activity ${ }^{7,8,31-35}$, and although most (but not all ${ }^{36}$ ) ncRNA genes are expressed at relative low levels, they may provide more information about cortical cell type identity than their protein-coding counterparts ${ }^{13,37}$.

Consistent with the observations mentioned above, ncRNAs have been implicated in brain aging and in the pathophysiology of neuropsychiatric and neurodegenerative disorders $5,12,13,38$. In this Review, we survey the emerging roles of ncRNAs in neurodegeneration and discuss in which ways they challenge our understanding of neurodegenerative diseases and how they might lead to novel diagnostic and therapeutic strategies.

\section{[H1] ncRNAs in CNS homeostasis}

During CNS development, ncRNAs affect stem cell maintenance, progenitor proliferation and cell fate choice ${ }^{5,13,19,25,27,39-41}$. miRNAs such as miR-124 and miR-132 have a marked positive regulatory impact on neurogenesis ${ }^{42,43}$, while some IncRNAs, such as rhabdomyosarcoma 2 associated transcript (RMST) and Tcl1 upstream neuron-associated long intergenic ncRNA (TUNA), induce neuronal differentiation ${ }^{17,20}$. Moreover, ncRNAs are implicated in neuronal fate commitment (for example miR-124 (REF. ${ }^{44}$ ), embryonic ventral forebrain 2 (Evf2) (REF. ${ }^{18}$ ), double-stranded neuron-restrictive silencer factor (dsNRSF) ${ }^{45}$ and distal-less homeobox 1, antisense ( $\mathrm{D} \mid \times 1 A S^{21}$ ), in glial specification (for example, miR-219 and miR-338 (REF. ${ }^{46}$ ), Sox8 opposite transcript (Sox8OT) and nuclear enriched abundant transcript 1 $(\text { NEAT1) })^{21}$, and NK2 homeobox $2(N k x 2.2)\left(\right.$ REF. $\left.^{22}\right)$ ) or in both (for example, miR-9 (REFs ${ }^{46,47}$ ) and Gomafu' ${ }^{21,35}$ ).

A broad array of distinct ncRNA classes - such as small ncRNAs (miRNAs ${ }^{48}$, endogenous small-interfering RNAs (endo-siRNAs), small nucleolar (sno)-derived RNAs ${ }^{33}$ and PIWI-interacting RNAs (piRNAs) ${ }^{16}$, long natural antisense transcripts (NATs) ${ }^{49,50}$ ), enhancer ncRNAs (eRNAs) ${ }^{32}$, circRNAs 7,8 and other IncRNAs ${ }^{51}$ (for example, metastasis associated lung adenocarcinoma transcript 1 (MALAT1) and NEAT1 (REF. ${ }^{31}$ ) and Gomafu ${ }^{35}$ ) - have been 
implicated in synaptic plasticity. Synaptically enriched ncRNAs, such as certain miRNA precursors, various miRNAs (for example, miR-9, miR-132, miR-134 and miR-138) ${ }^{52,53}, \mathrm{BC} 1$, $\mathrm{BC}_{200^{13}}$ and most brain circRNAs ${ }^{7,8}$, act as synaptic regulators in symphony with their protein-coding counterparts to regulate local protein expression ${ }^{54}$. A natural antisense transcript of $B D N F$ (BDNF-AS) may negatively regulate synaptic plasticity by repressing the transcription of $B D N F^{50}$, whereas another InCRNA, MALAT1, may be a positive regulator of synaptogenesis ${ }^{15}$.

Epigenetic and transcriptional changes at the synapse contribute to memory consolidation and storage. A growing body of evidence demonstrates that ncRNA-mediated regulation is instrumental for memory formation ${ }^{14,55,56}$. Small ncRNAs, including miRNAs ${ }^{14,56}$, piRNAs ${ }^{16,55}$ and $\mathrm{BC} 1$ (REF. ${ }^{56}$ ), are associated with cognitive and behavioural processes. Research on other ncRNA classes is still in its infancy. Importantly, knocking down an endogenous antisense ncRNA against $U b 3 a$, a gene imprinted in Angelman syndrome, rescues some of the cognitive deficits that are observed in a genetic mouse model of this disorder ${ }^{57}$. Although the exact regulatory impact of several identified ncRNAs on memory and cognition remains elusive, the available evidence suggests that ncRNA networks are important for CNS homeostasis and that their dysregulation could have profound consequences for brain function.

\section{[H1] ncRNA networks and neurotoxicity}

A number of distinct ncRNA classes are implicated in neurodegenerative disorders (Supplementary information S1 (table)). Aberrations in the transcriptional networks in which these ncRNAs operate (BOX 2) may impinge on brain homeostasis in complex ways ${ }^{58}$ (FIG. 2). This is well illustrated by studies of the C9ORF72-associated hexanucleotide repeat expansion (GGGGCC), the most common genetic cause of amyotrophic lateral sclerosis (ALS) and frontotemporal dementia (FTD). These repeat sequences are transcribed in both sense and antisense RNA and accumulate in nuclear and cytoplasmic RNA foci, whose number correlates with pathology severity in C9ORF72-related cases of ALS and FTD ${ }^{59-61}$. In addition, six dipeptide repeat proteins are translated from the C9ORF72 repeat locus (so-called repeat-associated non-ATG (RAN) translation) and co-aggregate in neuronal, ubiquitincontaining, intranuclear and cytoplasmic inclusions in the brain and spinal cord, possibly contributing to protein-mediated neurotoxicity mechanisms ${ }^{62,63}$ (FIG. 2).

Spinocerebellar ataxia type 8 (SCA8) is similarly caused by multiple transcriptional products of SCA8. The sense strand gives rise to a trinucleotide repeat expansion transcript and to a polyglutamine expansion protein, which accumulates in intranuclear inclusions in cerebellar and brainstem neurons of transgenic mice and human autopsy tissue ${ }^{64}$. Moreover, a pathogenic repeat sequence is transcribed in the antisense direction giving rise to a noncoding repeat RNA that overlaps with the Kelch-like protein 1 (KLHL1) gene (the ncRNA gene is known as SCA8, ataxin 8 (ATXN8) opposite strand (ATXN8OS) or KLHL1 antisense) ${ }^{64,65}$. Transgenic mice overexpressing the human SCA8 expansion sequence display cerebellar deficits and progressive motor deficits that are similar to those observed in affected individuals ${ }^{65}$. The accumulation of both sense and antisense expansion transcripts, and of a polyglutamine expansion protein in these mice, suggests that SCA8 pathology possibly involves both protein and RNA gain-of-function mechanisms ${ }^{65}$. 
Non-protein coding genes may also have roles alongside protein-coding counterparts in fragile $X$ syndrome (FXS), Huntington's disease (HD) and Alzheimer's disease (AD). The expansion of the microsatellite locus in the fragile $X$ mental retardation 1 (FMR1) gene is associated with FXS (>200 CGG repeats; termed full mutation) and the related condition fragile X-associated tremor and ataxia syndrome (FXTA) (55-200 CGG repeats; termed premutation). This locus gives rise to four distinct transcripts with possible pathogenic relevance: the FMR1 repeat-containing mRNA; the FMR5 RNA, a sense ncRNA that is transcribed upstream of the FMR1 promoter; the FMR6 RNA, an antisense ncRNA that overlaps the FMR1 3'-untranslated region (UTR); and, finally, FMR4, an antisense transcript from FMR1 that spans the repeat region ${ }^{66,67}$ (FIG. 2). These transcripts show different expression patterns in the brains of individuals carrying the premutation or the full mutation.

This complex transcriptional fingerprint may contribute to the variability of the clinical phenotypes observed in FXTAS and FXS ${ }^{66,67}$. Transcriptional silencing of FMR1 in patients with FXS leads to a deficit in its protein product FMRP, an RNA-binding protein that regulates local protein translation in dendrites. Fmr1 knockout mice display synaptic alterations and cognitive impairment, suggesting a causal link between FMRP loss-offunction and FXS pathology ${ }^{68}$. However, FMR1 expansion repeat RNA co-localizes with ubiquitin in intranuclear inclusions in postmortem FXTAS brain ${ }^{67}$, and FMR4, which is also silenced in individuals with FXS, exerts antiapoptotic functions in human cell lines ${ }^{69,70}$. These findings illustrate the complexity of the gain- and loss-of-function mechanisms in brain disorders such as FXTAS and FXS.

In HD, a CAG repeat expansion in exon 1 of the gene huntingtin (HTT) is the primary cause of pathology. A small sense repeat transcript (sCAG) derived from the repeatcontaining mRNA is elevated in the brains of individuals with $\mathrm{HD}$ and is neurotoxic in vitro ${ }^{71}$, indicating that it might contribute to HD pathology. However, an antisense ncRNA overlapping the HTT repeat locus (HTT-AS) acts as an HTT repressor and is downregulated in brain tissue from patients with $\mathrm{HD}^{72}$. Since lowering mutated and wild-type $\mathrm{HTT}$ levels ameliorates pathology in HD mice ${ }^{73}$, HTT-AS should have a protective role in the disorder.

In $A D$, the evidence for a role of cross-talking coding and non coding transcriptional networks is much less straightforward. Single nucleotide polymorphisms (SNPs) in the vicinity of a locus giving rise to both antisense ((antisense non-coding RNA in the INK4 locus (ANRIL; also known as CDKN2BAS) and circular (CANRIL) transcripts, which have been reported to be involved in epigenetic regulation, have been associated with $A D$ pathology $y^{74-}$ 77. These associations have not yet been confirmed in larger, classic genome-wide association studies (GWAS), but the observations may be of interest as CDKN2B, which gives rise to these transcripts, encodes a protein involved in cell cycle regulation that accumulates in neurofibrillary tangles and amyloid plaques, which are pathological features observed in the brains of patients with $A D^{78}$. In addition, an antisense transcript of the glia-derived neurotrophic factor (GDNF) gene (GDNFOS), gives rise - via alternative splicing - to two ncRNAs and one protein and has been implicated in aberrant GDNF mRNA splicing in human $A D$ brain tissue ${ }^{79}$. The functional consequences of these events for the physiological neurotrophic and neuroprotective functions of GDNF and for AD pathogenesis remain, however, unclear. 


\section{[H1] ncRNA mechanisms in neurodegeneration}

A growing list of studies illustrates the diversity of ncRNA roles in brain function and dysfunction (Supplementary information S1 (table)). Gaining a deeper understanding of ncRNA-mediated mechanisms of regulation will eventually facilitate their efficient therapeutic targeting. Here, we summarize six major mechanisms that likely contribute to the neurodegenerative process (TABLE 1 and FIG. 3).

[H3] Epigenetic regulation. Chromatin immunoprecipitation (ChIP) assays demonstrate that ncRNAs with links to neurodegeneration associate with chromatin remodeling complexes and may therefore play roles in epigenetic regulation.

An example is a ncRNA associated with SCA7, where polyglutamine repeat expansions in ATXN7 cause neurodegeneration(REF. ${ }^{80}$ ). . Convergent transcription of spinocerebellar ataxia-7 antisense noncoding transcript 1 (SCAANT1) suppresses Atxn7 transcription in the sense direction in mice ${ }^{80}$. SCAANT1 levels inversely correlate with ATXN7 mRNA levels in fibroblasts from patients with SCA7 and in transgenic mice carrying the repeat disease locus ${ }^{80}$. Hence, a feedforward regulation explanation has been proposed, wherein the repeat expansion in ATXN7 reduces SCAANT1 expression, which leads to derepression of $A T X N 7$ transcription and increased mutant ATXN7 levels ${ }^{80}$.

The IncRNAs BDNF-AS, TUG1, MEG3, NEAT1 and TUNA, which may act as epigenetic regulators, are differentially expressed in brain tissue from humans with HD versus that from healthy individuals ${ }^{17,81-83}$ and might be involved in the pathological outcome by modifying the effects of mutant HTT. In particular, BDNF-AS, which is activity-dependent, acts as a scaffold to recruit polycomb repressive complex 2 (PRC2) to the BDNF promoter, resulting in $B D N F$ transcriptional repression in a human cell line ${ }^{31,50}$. This regulatory effect may be of relevance in $\mathrm{HD}$, in which $B D N F$ is downregulated in the human brain ${ }^{84}$. Interestingly, BDNF overexpression rescues dopaminergic, synaptic, motor and cognitive deficits in a HD transgenic mouse model ${ }^{82}$, and BDNF-AS inhibition provides neuroprotection upon ischemic insult in retinal ganglion cells ${ }^{85}$. Importantly, the regulatory effects of all the differentially expressed IncRNAs mentioned above still await systematic experimental validation in HD models ${ }^{17,81}$

[H3] RNA interference. Posttranscriptional repression of gene expression by miRNAs and small-interfering RNAs (siRNAs) is collectively termed RNA interference (RNAi). Key molecular components shared between miRNA- and siRNA-mediated mechanisms are the RNase III endonuclease Dicer, which has a role in the generation of miRNAs and siRNAs, and the argonaute (AGO) protein family, which is a core component of the RNA-induced silencing ribonucleoprotein complex (RISC) that brings together the ncRNA and the mRNA target ${ }^{86}$. miRNAs bind to their targets leading to either mRNA decay or translational inhibition. Global or individual manipulation of miRNA levels in the rodent brain can lead to neurodegenerative phenotypes ${ }^{26,87-89}$. Indeed, genetic ablation of Dicer in adult mouse brain promotes hyperphosphorylation of TAU and neuronal loss in the hippocampus ${ }^{26}$, which are both features of $A D$, whereas increasing the levels of the miRNA let-7 induces neuronal death in the mouse cortex ${ }^{88}$. miRNA expression profiles are perturbed across a wide spectrum of neurodegenerative disorders, including AD, Parkinson's disease (PD), HD, ALS, 
FXS, FTD and SCA (Supplementary information S1 (table)). miRNA-mediated regulatory networks involve multiple targets and therefore altered levels of one miRNA in the CNS might impact several layers of cellular homeostasis ${ }^{90}$. This is illustrated in knockdown experiments of miR-132, which is consistently and robustly downregulated in AD brain tissue ${ }^{90,91}$. In $A D$ transgenic mice, miR-132 downregulation in the hippocampus promotes both the accumulation of amyloid- $\beta$ (a hallmark of AD) and TAU hyperphosphorylation by upregulating inositol-trisphosphate 3-kinase $\mathrm{B}^{92}$. In addition, miR-132 inhibition in primary cortical and hippocampal neurons in vitro,, leads to the activation of the forkhead box protein 03 (FOXO3)-PTEN-P300 signalling pathway, which induces neuronal death ${ }^{93}$.

Other ncRNAs may also act via the RNAi pathway. The small neurotoxic repeatcontaining transcript SCAG, which stems from HTT and is upregulated in the frontal cortex and caudate of brains from individuals with HD and in brain tissue from HD transgenic mice, is loaded into the RISC to act as a gene silencer ${ }^{71}$. Interestingly, sCAG RNAs isolated from cells expressing human mutant HTT and from HD-affected human brain tissue markedly decreased the viability of human neuronal cells via an AGO2-dependent mechanism ${ }^{71}$. Antisense oligonucleotides against sCAGs reversed this effect, supporting a role for sCAGs in mutant HTT-mediated neurotoxicity ${ }^{71}$. Conversely, the antisense ncRNA derived from the same HTT locus (HTT-AS) seems to repress HTT expression also via a Dicer-dependent regulatory mechanism. HTT-AS is downregulated in human HD brain tissue ${ }^{72}$. An interesting model emerges from these observations in which the presence of the repeat expansion in the HTT-AS transcript represses its transcription, which may result in elevated mutant HTT levels in HD.

[H3] Alternative splicing. Shifting the splicing profiles of transcripts is another mechanism by which ncRNAs may affect pathology in neurodegenerative disorders. Sortilin-related receptor 1 (SORL1) is a risk gene for late-onset $A D$ that regulates the trafficking of amyloid- $\beta$ precursor protein (APP) - from which amyloid- $\beta$ is derived - and can be proteolytically processed by $\beta$-site APP cleaving enzyme 1 (BACE1; also known as $\beta$-secretase 1 ), which is involved in amyloid- $\beta$ generation ${ }^{94}$. SORL1 levels are decreased in the brains of individuals with $A D^{94}$, and Sorl1 ablation in mice induces amyloid- $\beta$ formation by shifting APP from the retromer recycling pathway to the BACE1 proteolytic pathway ${ }^{95}$. An antisense transcript termed SORL1-AS (also known as 51A) shifts splicing so that transcripts for the alternative $B$ and $\mathrm{F}$ isoforms of SORL1 are produced over the transcript for the canonical, long SORL1 isoform A, leading to downregulation of canonical SORL1. This shift in splicing is associated with increasing amyloid- $\beta$ levels in cultured human neuronal cells ${ }^{94}$. Notably, SORL1-AS is upregulated in human $A D$ cortex, suggesting that it might contribute to the SORL1 deficit and elevated amyloid- $\beta$ production that are observed in the disease.

Another ncRNA that may be involved in $A D$, at least in part, through alternative splicing is the IncRNA 17A. This ncRNA is also upregulated in AD brain tissue ${ }^{96}$ and may have a dual impact, as it increases amyloid- $\beta$ generation and induces alternative splicing of $G A B A B 2$, which encodes $G_{A B A_{B}}$ receptor subunit 2, abolishing signalling mediated by receptors containing this subunit in neuroblastoma cells in vitro ${ }^{96}$.

In SCA8, ATXN8OS accumulates in RNA foci in the brain ${ }^{97}$. ATXN8OS promotes alternative splicing of the sodium- and chloride-dependent GABA transporter 4 gene (Gabt4) and increased expression of the encoded protein in SCA8 transgenic mice ${ }^{97}$, and such 
changes are also observed in autopsy-obtained brain tissue from patients with SCA $8^{97}$. Increased GABT4 levels have been linked to a reduction of GABA at synapses in cerebellar granular neurons ${ }^{97}$. In SCA8 transgenic mice, ATXN8OS was implicated in loss of GABAergic inhibition in the cerebellar granular cell layer and induction of a progressive motor phenotype, linking a RNA gain-of-function mechanism directly to SCA8 pathology ${ }^{97}$. ATXN8OS might also regulate the splicing of its sense transcript, the mRNA for KLHL165, although the relevance of this effect to disease pathology is unknown.

Finally, the widespread alterations in FMR1 splicing profiles in the brain in FXS suggest that FMR6, a natural antisense FMR1 transcript that is downregulated in FXS brains, regulates the canonical splicing of FMR1 (in addition to other effects) ${ }^{66}$. The functional validation of these observations may provide further insights into disease pathology.

[H3] mRNA stability. Cytoplasmic RNA-RNA duplex formation between naturally occurring antisense and cognate sense transcripts can prevent endo- or exonucleolytic degradation of the sense mRNA, or block access to proteins involved in RNA turnover, increasing its stability and translation ${ }^{98}$. For example, the BACE1 antisense ncRNA (BACE1-AS) binds BACE1 mRNA, leading to increased BACE1 levels in human cell lines and mouse brain. As indicated above, BACE1 is one of the proteases that generate amyloid- $\beta$, and it has become a major drug target that is being currently explored in phase 3 clinical trials for AD. BACE1-AS is elevated in the brains of individuals with $A D$ and of some transgenic mouse models of the disease ${ }^{99}$. Moreover, it is increased upon exposure to amyloid- $\beta$ in vitro ${ }^{99}$, suggesting that a possibly deleterious positive feedback loop involving BACE1-AS and amyloid- $\beta$ may be maintained during $A D$ progression.

In contrast to BACE1-AS, ciRS-7 (also known as CDR1-AS) is a strongly brain-enriched circular antisense ncRNA ${ }^{100}$ that is downregulated in the cortex and hippocampus of patients with sporadic $A D^{101}$. This ncRNA has mainly been studied for its role as a molecular decoy, which is discussed below. However, ciRS-7 also stabilizes - via an as yet unknown mechanism - its sense transcript, the mRNA for cerebellar degeneration-related antigen 1 (CDR1), which was first identified in patients with paraneoplastic cerebellar degeneration ${ }^{100}$. Of note, one preliminary report suggested that CDR1 might be increased in lymphocytes from patients with $A D$, but the importance of this observation for $A D$ pathology in the human brain remains unclear ${ }^{102}$.

Another example of a potentially disease-linked, mRNA stability-related mechanism concerns an antisense transcript from the PTEN-induced putative kinase 1 (PINK1) locus (PINK1-AS). Mutations in PINK1 are causally related to $P D^{103}$, and PINK1-AS positively regulates the abundance of a specific splice variant of PINK1 (sVPINK1) in neuroblastoma cells, possibly via RNA-RNA hybridization and transcript stabilization ${ }^{104}$.

Last, FMR6, which has already been discussed above, might stabilize FMR1 mRNA ${ }^{66}$. The findings relating to FMR6 and indeed PINK-AS, suggest that this ncRNA-mediated regulation of disease-associated protein-coding transcripts might theoretically impact the activity of the related proteins and thereby modify disease progression, although this hypothesisrequires further experimental validation in disease models.

[H3] Translational regulation. Certain ncRNAs have a direct impact on the translation of mRNA transcripts. BC200, a small neuron-specific ncRNA, is transported in ribonucleoprotein 
particles to the dendrites where it forms part of a complex with multiple protein interactors (including FMRP) that regulates the translation of several mRNAs in mouse brain ${ }^{105}$. Given that local translation in dendrites is involved in long-term synaptic plasticity and that BC200 levels markedly increase in human $A D$ brain, it is intriguing to hypothesize that $B C 200$ might be implicated in the synaptic defects that are observed in AD ${ }^{106}$.

Ubiquitin carboxyl-terminal hydrolase isozyme L1 (UCHL1) is a brain-enriched protein that is highly expressed in the substantia nigra and that is involved in dopaminergic neuron differentiation and in the ubiquitin-proteasome system. GWAS indicate that UCHL1 is a PD risk gene ${ }^{107}$. An antisense transcript for this gene (UCHL1-AS) targets Uch/1 mRNA to heavy polysomes for translation, resulting in increased UCHL1 levels ${ }^{108}$. In in vitro PD models, both UCHL1-AS and Uchl1 mRNA are downregulated, suggesting a possible link to the proteasomal deficits that are observed in $\mathrm{PD}^{109}$.

[H3] Molecular decoys. Owing to their highly versatile, modular nature, ncRNAs can act as molecular traps that titrate away an RNA (in this context the ncRNAs are called competing endogenous RNAs (ceRNAs) ${ }^{110}$ ) or a protein target ${ }^{111}$, or transport them to specific cellular compartments. The relative abundance of the ncRNA decoy and of its targets along with the number of target molecules that can be sequestered by one single ncRNA in a given cellular context are critical factors that determine the outcome of such interactions ${ }^{112,113}$. More specifically, the concentration of the ceRNA must approach that of the target miRNA to induce meaningful de-repression of the miRNA targets ${ }^{113,114}$.

The hexanucleotide repeat-containing C9ORF72 sense transcript, which is involved in FTD and ALS, co-accumulates with the antisense C9ORF72 ncRNA in nuclear and cytoplasmic RNA foci in human cultured cells and in patient brain tissue ${ }^{60,63}$. Several RNA-binding proteins can be sequestered in these foci in brain tissue from humans with FTD or ALS and from mice with this expansion mutation, leading to altered RNA splicing or perturbed nucleocytoplasmic transport and possibly contributing to certain aspects of the pathology63,115.

The ncRNAs MALAT1 and NEAT1 are elevated in the brain of patients with FTLD-TDP, where they are the two most highly detected RNAs in TAR DNA-binding protein 43 (TDP43)positive disease-associated inclusions ${ }^{116}$. In mammalian cells, MALAT1 and NEAT1 are implicated in recruiting splicing factors to subnuclear bodies called speckles and paraspeckles, respectively ${ }^{116,117}$. These observations clearly suggest that splicing aberrations contribute to disease, although the extent to which they influence the progression of pathology needs further assessment. This is also the case for the antiapoptotic transcript FMR4 (the antisense transcript of FMR1), which is highly expressed in individuals with FXTAS and silenced in FXS, and sequesters RNA-binding proteins into intranuclear inclusions in cultured human cells ${ }^{67,69}$.

The antisense ncRNA LRP1-AS can act as a molecular decoy for the ubiquitous chromatin-associated protein HMGB2, serving as a cell type- and locus-specific natural RNA ligand to fine tune HMGB2 activity in vitro ${ }^{118}$. This interaction obscures the binding of HMGB2 to its target LRP1, resulting in reduced Lrp1 transcription. Interestingly, LRP1-AS is upregulated in the superior frontal gyrus in brain tissue from patients with $A D$ and its levels are inversely correlated with LRP1 levels ${ }^{118}$. Although cell biology studies suggest a role for 
LRP1 in amyloid- $\beta$ metabolism in brain, the relevance of LRP1 regulation by LRP1-AS in AD pathology remains to be clarified.

The aforementioned circular RNA ciRS-7 represents a unique example of multifunctionality. Notably, ciRS-7 is expressed at 200-fold higher levels than housekeeping proteins in human and mouse brains ${ }^{100,114}$, suggesting that ciRS-7-mediated molecular trapping mechanisms in CNS are stoichiometrically relevant. Apart from stabilizing CDR1 mRNA, as discussed above, human ciRS-7 harbors 74 seed sequence matches for miR-7 (which reflects a 10-fold higher miRNA-binding capacity than any other known transcript) and acts therefore as a miR-7 'sponge' in neuronal tissues ${ }^{114,119}$. In brains from AD patients, decreased ciRS-7 levels in the hippocampus and the cortex are associated with miR-7 upregulation and consequent repression of miR-7 targets involved in ubiquitin-mediated clearance of amyloid- $\beta^{101}$. In vitro, ciRS-7 promotes APP and BACE1 degradation by both the proteasomal and the lysosomal pathways, leading to decreased amyloid- $\beta$ levels ${ }^{120}$. Hence, a complex link is emerging between ciRS-7 deficits and pathological amyloid- $\beta$ aggregation in the AD brain. Moreover, since one of the targets of miR-7 is $\alpha$-synuclein mRNA, which is encoded by SNCA, this regulatory network might have a role in PD as well ${ }^{121}$ ( $\alpha$-synuclein is implicated in the pathophysiology of PD). Interestingly, endogenous $\alpha$-synuclein mRNA levels decrease upon transfection of a human cell line with miR-7, a change that is counteracted by overexpression of ciRS-7 (REF. ${ }^{114}$ ). ciRS-7 is degraded in a miR-671-dependent manner (possibly following direct ciRS-7-miR-671 base pairing) via AGO2-mediated cleavage in a human cell line ${ }^{100}$, suggesting that ciRS-7 acts to transport a cargo of miR-7 that becomes released by miR-671. Such spatiotemporal control of miR-7 activity in the cell might go astray in neurodegeneration.

Finally, Inc-SCA7 acts as a miRNA decoy to regulate ATXN7 mRNA in a brain regiondependent manner ${ }^{122}$. More specifically, Inc-SCA7 competes with the ATXN7 transcript for binding to miR-124, thereby increasing ATXN7 levels in mouse and human neuroblastoma cells. Further experimental confirmation of these findings is required, as disruption of this tripartite regulation might play a role in SCA7 pathology, in which the levels of expansion repeat-containing ATXN7 are elevated ${ }^{122}$.

\section{[H1] Genetic associations}

Only $7 \%$ of the nearly 6,500 disease- or trait-predisposing SNPS that have been identified in more than 1,200 GWAS over the past decade are located in protein-coding regions, suggesting that most of these GWAS-associated SNPs regulate gene expression rather than altering the protein sequence or structure ${ }^{123}$. Interestingly, $75 \%$ of the SNPs that affect lincRNA expression do so in a tissue-dependent manner and without influencing the expression of neighbouring protein-coding genes ${ }^{123}$. Nevertheless, hard genetic proof for a causal role of SNPs in ncRNA transcripts or ncRNA-interacting genomic loci in neurodegeneration is lacking (Supplementary information S2 (table)). For instance, a polymorphism in the $3^{\prime}$-UTR of oxidized low density lipoprotein receptor 1 (OLR1) mRNA may impair amyloid- $\beta$ clearance from the brain across the blood-brain barrier (BBB) and was associated with cerebral amyloid angiopathy in a small cohort of patients with $A D^{124}$. Moreover, a variation in the miR-433-binding site of FGF2O induces the expression of both FGF20 and $\alpha$-synuclein and is associated with increased risk for PD ${ }^{125}$. 
FTLD-TDP can be caused by loss-of-function mutations in progranulin (GRN). A genetic variant in a binding site for miR-659 in the 3'-UTR of GRN mRNA increases the binding of the miRNA to its target, resulting in stronger $G R N$ repression; this variant has been reported by some studies to be a susceptibility factor for FTLD-TDP, AD and hippocampal sclerosis, although these findings require further confirmation by independent reports ${ }^{126-130}$. In another small-sized study, a similar polymorphism in one of miR-146a-5p precursors was associated with a genetic predisposition for $A D^{131}$. This polymorphism represses miR-146a-5p expression and consequently de-represses its target $T L R 2$, which has been functionally implicated in amyloid- $\beta$-dependent inflammatory signalling cascades ${ }^{131}$.

Recent findings suggest that SNPs in noncoding enhancer regions that are in close proximity to disease-associated loci (for example, SNPs in the SNCA locus, which is linked to PD risk) may potentially affect gene expression in an organ-specific fashion, resulting in distinct disease phenotypes ${ }^{132,133}$. Limited knowledge about the key functional elements in genes coding for ncRNAs and the rules of interaction among them, and the overall lack of conservation of ncRNAs across different species obstruct the functional validation of genetic variations in these loci ${ }^{134}$. Indeed, developing an understanding of the molecular mechanisms governing ncRNA involvement in disease is dauntingly hard, as SNPs in ncRNA genes could alter ncRNA expression or structure, and/or their functional interactions with DNA, RNA or protein partners.

\section{[H1] ncRNA diagnostics and therapeutics}

[H3] ncRNAs as biomarkers in neurodegenerative disorders. Alterations in miRNA levels in cerebrospinal fluid (CSF) and peripheral tissues in neurodegenerative disorders have been extensively documented ${ }^{135-143}$. Although it is clear that miRNAs are markedly dysregulated in various neurodegenerative processes, most studies in this area have been monocentric and relatively limited with regard to sample size. Differences between studies in standardization of sample stratification, collection, processing, data normalization and analysis ${ }^{136}$ explain why these reports have not yet yielded a consensus on which of the altered miRNAs are relevant to disease. For instance, postmortem delays in sample collection can be crucial, as miRNA levels in postmortem CSF may not correlate well with those in brain owing to compromised BBB integrity, which can result in the rapid entry of brain miRNAs in CSF $^{137}$. The need for systematic and standardized approaches to profile circulating miRNAs in CSF and blood is evident.

Recent findings suggest that miR-206 levels (upregulated in the brain in AD) can be measured in olfactory mucosa in patients with mild cognitive impairment and that the expression of this miRNA correlates with the degree of cognitive deficit. These observations could prove exciting in the context of the early diagnosis of $A D$, as this approach may allow access to the molecular changes occurring in living cells ${ }^{144}$. It should be noted, however, that only 41 patients were investigated in this monocentric study, so further confirmation of these results is needed ${ }^{144}$. In patients with ALS or FTD, sense and antisense C9ORF72 RNA foci have been found in fibroblasts and lymphoblasts ${ }^{61,62}$, and polyglutamine proteins have been detected in CSF $^{145}$, suggesting the potential of these RNA and protein species as possible biomarkers. Again the relative low number of cases investigated (fewer than 10 
cases per study) makes it imperative to repeat these studies in much larger patient cohorts to gain confidence in their diagnostic potential.

Although ncRNAs seem to be quite stable in body fluids (owing to their secondary structures) $)^{1,7}$, further study is warranted to evaluate their potential as neurodegeneration biomarkers ${ }^{146}$. Efforts to set up large, multicentric and well-controlled studies to document ncRNA alterations in CSF and blood in a systematic way are necessary ${ }^{147}$.

[H3] Novel ncRNA-based therapies in neurodegeneration. Targeting ncRNAs might offer effective approaches for the treatment of neurodegenerative disorders ${ }^{12}$. Successful targeting of IncRNAs will presumably be difficult because of their extensive secondary structures ${ }^{146}$; however, improved oligonucleotide design has delivered multiple chemically modified analogues that may overcome such limitations ${ }^{148}$, and several of these tools have been successfully employed in experimental models of neurodegeneration ${ }^{149}$. Antisense oligonucleotides (ASOs) against the repeat-containing C9ORF72 transcripts and small molecules that inhibit RNA translation suppressed RNA foci formation in patient fibroblasts and in neurons derived from induced pluripotent stem cells from individuals with ALS. In C9ORF72 repeat-overexpressing mice, ASOs improved cognitive deficits ${ }^{61,145,150-153}$. Furthermore, cleavage (siRNA) or inhibition (antagoNAT) antisense strategies against NATs potentially involved in $A D$ and $H D$ modulated the levels of both the NAT and its cognate sense mRNA in mouse and human cell lines and in mouse brain ${ }^{50,99,148}$. Interestingly, inhibition of BACE1-AS lowered amyloid- $\beta$ levels and improved adult neurogenesis in a mouse model of $A D^{154}$, and an antagoNAT against BDNF-AS increased endogenous BDNF levels and promoted neuronal growth and survival in wild-type murine brain ${ }^{50}$. Finally, emerging evidence suggests that blocking the generation of certain circRNAs may counteract TDP43-mediated cytotoxicity, and this was suggested as a potential therapeutic strategy for $\mathrm{ALS}^{155}$.

A major advantage of single-stranded oligonucleotides, such as antagoNATs and ASOs, is that they can be administered systemically as 'naked' molecules (that is, without the requirement for any delivery vehicles) ${ }^{148}$. Direct administration of oligonucleotides to the CNS has been achieved via intracerebroventricular or intrathecal infusion of CSF into rodents and non human primates in tauopathy, HD, ALS and spinal muscular atrophy (SMA) animal models ${ }^{73,148,156-160}$. These approaches demonstrated that ASOs delivered in CSF efficiently enter the brain, where they engage their RNA targets leading to up- or downregulation of the targeted transcripts or to shifts in splicing profiles and, eventually, to the amelioration of tissue toxicity and cognitive deficits.

Other emerging strategies for targeting the CNS include nanotechnology-based drug delivery systems. For instance, exosomes, which are nano-vesicles of endocytic origin, efficiently mediate the intercellular transfer of siRNAs, miRNAs and miRNA antisense oligonucleotides in vitro ${ }^{161-163}$. In vivo, intravenous delivery of autologous dendritic cellderived exosomes engineered for neuronal targeting and loaded with an siRNA against BACE1 resulted in marked brain-specific BACE1 mRNA and protein knockdown and a reduction in amyloid- $\beta$ in the cortex of treated mice ${ }^{164}$. The efficient and tissue-specific delivery along with the absence of overall immune responses underline the potential for using such RNA-based systemic therapeutic approaches in chronic neurodegenerative conditions. 
These findings have not gone unnoticed by the pharmaceutical industry and several companies are now focusing on ncRNAs (miRNAs, NATs and IncRNAs) as potential treatments for various neurological disorders ${ }^{146,165}$. Most of the practical attempts are still in the lead optimization phase, with only a few (against hepatitis C, lymphoma and fibrosis) having reached Phase 1 and 2 clinical trials. Apart from the great hurdle of efficiently crossing the BBB (which is common to all CNS-targeting drugs), ncRNA-based therapeutics are additionally facing the issue of the differential targeting of the multiple transcriptional products generated by the same target locus and the associated complex biology. Nevertheless, clinical trials in patients with ALS or SMA that involve intrathecal delivery of ASOs against protein-coding transcripts have demonstrated the promise of therapeutically targeting RNA ${ }^{166,167}$. Most notably, infants with type 1 SMA (the most severe form of the disease) that were enrolled in a recent Phase 3 clinical trial showed markedly improved motor function following treatment with an ASO that interferes with the splicing of the transcript encoding SMN2, thereby boosting the levels of the SMN2 protein ${ }^{167}$.

\section{[H1] Perspectives}

Despite substantial progress in understanding ncRNA biology and its contribution to disease, we can safely state that we are in the early days of this field. The vast majority of the annotated ncRNAs have not been functionally investigated and many questions remain unanswered regarding the impact of ncRNAs in the context of neurodegeneration. The only way forward is to perform more basic research to address the role of different ncRNAs in the brain, in specific brain areas, and in distinct cells. Larger, higher-powered and bettercontrolled ncRNA-profiling screens in human patients in CSF, blood and, indeed, brain are needed to map the changes in ncRNAs in disease with greater confidence ${ }^{147}$. In the meantime, novel insights, regarding mitochondrially encoded ncRNAs, ncRNA editing (epitranscriptomics) and epigenetic regulation of ncDNA, continue emerging 40,168,169. Systems biology and bioinformatic approaches are necessary to unravel the highly intricate networks in which ncRNAs operate ${ }^{170,171}$. This will also require the implementation of novel experimental approaches, such as the currently rapidly evolving technologies to screen the transcriptome at the single-cell level ${ }^{37}$ or in different spatial contexts ${ }^{172}$. Looking ahead to the next decade, we anticipate that valuable insights will be gained into how these fascinating molecules contribute to hitherto unknown aspects of pathogenic mechanisms and how they may be critical to understanding the human-specific aspects of neurological diseases (BOX 3). Finally, we will hopefully witness impovements in the diagnosis and treatment of neurodegenerative disorders resulting from our developing understanding of ncRNAs. 
1. Rinn, J. L. \& Chang, H. Y. Genome regulation by long noncoding RNAs. Annu. Rev. Biochem. 81, 145-166 (2012).

2. Morris, K. V \& Mattick, J. S. The rise of regulatory RNA. Nature reviews. Genetics 15, 423-37 (2014).

3. Kadakkuzha, B. M. et al. Transcriptome analyses of adult mouse brain reveal enrichment of IncRNAs in specific brain regions and neuronal populations. Front. Cell. Neurosci. 9, (2015).

4. Webb, A. et al. RNA sequencing of transcriptomes in human brain regions: proteincoding and non-coding RNAs, isoforms and alleles. BMC Genomics 16, 990 (2015).

5. Salta, E. \& De Strooper, B. Non-coding RNAs with essential roles in neurodegenerative disorders. Lancet Neurol. 11, 189-200 (2012).

6. Smalheiser, N. R. et al. Natural antisense transcripts are co-expressed with sense mRNAs in synaptoneurosomes of adult mouse forebrain. Neurosci. Res. 62, 236-9 (2008).

7. Rybak-Wolf, A. et al. Circular RNAs in the Mammalian Brain Are Highly Abundant, Conserved, and Dynamically Expressed. Mol. Cell 58, 870-85 (2015).

One of the first reports on circular RNA functionality in mamallian brain.

8. You, X. et al. Neural circular RNAs are derived from synaptic genes and regulated by development and plasticity. Nat. Neurosci. 18, 603-10 (2015).

One of the first reports on a circular RNA role in synaptic plasticity.

9. Derrien, T. et al. The GENCODE v7 catalog of human long noncoding RNAs: analysis of their gene structure, evolution, and expression. Genome Res. 22, 1775-89 (2012).

10. Harrow, J. et al. GENCODE: The reference human genome annotation for The ENCODE Project. Genome Res. 22, 1760-1774 (2012).

11. Mehler, M. F. \& Mattick, J. S. Non-coding RNAs in the nervous system. J Physiol 5752, 333-341 (2006).

12. Qureshi, I. A. \& Mehler, M. F. Long Non-coding RNAs: Novel Targets for Nervous System Disease Diagnosis and Therapy. Neurotherapeutics 10, 632-646 (2013).

13. Briggs, J. A., Wolvetang, E. J., Mattick, J. S., Rinn, J. L. \& Barry, G. Mechanisms of Long Non-coding RNAs in Mammalian Nervous System Development, Plasticity, Disease, and Evolution. Neuron 88, 861-877 (2015).

14. Mercer, T. R. et al. Noncoding RNAs in Long-Term Memory Formation. Neuroscientist 14, 434-445 (2008).

15. Bernard, D. et al. A long nuclear-retained non-coding RNA regulates synaptogenesis by modulating gene expression. EMBO J. 29, 3082-3093 (2010).

16. Rajasethupathy, P. et al. HHMI Author Manuscript A role for neuronal piRNAs in the epigenetic control of memory- related synaptic plasticity. 149, 693-707 (2012).

17. Lin, N. et al. An evolutionarily conserved long noncoding RNA TUNA controls pluripotency and neural lineage commitment. Mol. Cell 53, 1005-19 (2014).

18. Bond, A. M. et al. Balanced gene regulation by an embryonic brain ncRNA is critical for adult hippocampal GABA circuitry. Nat. Neurosci. 12, 1020-1027 (2009).

19. Onoguchi, M. M., Hirabayashi, Y. Y., Koseki, H. H. \& Gotoh, Y. Y. A noncoding RNA regulates the neurogenin1 gene locus during mouse neocortical development. Proc. Natl. Acad. Sci. U. S. A. 109, 16939-16944 (2012).

20. Ng, S.-Y., Johnson, R. \& Stanton, L. W. Human long non-coding RNAs promote pluripotency and neuronal differentiation by association with chromatin modifiers and transcription factors. EMBO J. 31, 522-33 (2012).

21. Mercer, T. R. et al. Long noncoding RNAs in neuronal-glial fate specification and oligodendrocyte lineage maturation. BMC Neurosci. 11, 14 (2010).

22. Tochitani, S. \& Hayashizaki, Y. Nkx2.2 antisense RNA overexpression enhanced 
oligodendrocytic differentiation. Biochem. Biophys. Res. Commun. 372, 691-696 (2008).

23. Rani, N. et al. A Primate IncRNA Mediates Notch Signaling during Neuronal Development by Sequestering miRNA. Neuron 90, 1174-1188 (2016).

24. Sauvageau, M. et al. Multiple knockout mouse models reveal lincRNAs are required for life and brain development. Elife 2013, 1-24 (2013).

This paper provides a solid in vivo proof of concept for the functionality of lincRNAs in CNS.

25. Fatica, A. \& Bozzoni, I. Long non-coding RNAs: new players in cell differentiation and development. Nat Rev Genet 15, 7-21 (2014).

26. Hébert, S. S. et al. Genetic ablation of Dicer in adult forebrain neurons results in abnormal tau hyperphosphorylation and neurodegeneration. Hum. Mol. Genet. 19, 3959-69 (2010).

This is the first paper providing a causal link between microRNAs and hippocampal neurodegeneration.

27. Guttman, M. et al. lincRNAs act in the circuitry controlling pluripotency and differentiation. Nature 477, 295-300 (2011).

28. Goff, L. A. et al. Spatiotemporal expression and transcriptional perturbations by long noncoding RNAs in the mouse brain. Proc. Natl. Acad. Sci. U. S. A. 112, 6855-62 (2015).

29. Mercer, T. R., Dinger, M. E., Sunkin, S. M., Mehler, M. F. \& Mattick, J. S. Specific expression of long noncoding RNAs in the mouse brain. Proc. Natl. Acad. Sci. U. S. A. 105, 716-21 (2008).

This is the first report on expression specificity of long ncRNAs in the mammalian brain.

30. Belgard, T. G. et al. A transcriptomic atlas of mouse neocortical layers. Neuron 71, 605-616 (2011).

31. Lipovich, L. et al. Activity-dependent human brain coding/noncoding gene regulatory networks. Genetics 192, 1133-1148 (2012).

32. Kim, T. et al. Widespread transcription at neuronal activity-regulated enhancers. Nature 465, 182-7 (2010).

33. Smalheiser, N. R., Lugli, G., Thimmapuram, J., Cook, E. H. \& Larson, J. Endogenous siRNAs and noncoding RNA-derived small RNAs are expressed in adult mouse hippocampus and are up-regulated in olfactory discrimination training. RNA 17, 166181 (2011).

34. Mukilan, M., Ragu Varman, D., Sudhakar, S. \& Rajan, K. E. Activity-dependent expression of miR-132 regulates immediate-early gene induction during olfactory learning in the greater short-nosed fruit bat, Cynopterus sphinx. Neurobiol. Learn. Mem. 120, 41-51 (2015).

35. Barry, G. et al. The long non-coding RNA Gomafu is acutely regulated in response to neuronal activation and involved in schizophrenia-associated alternative splicing. Mol. Psychiatry 19, 486-494 (2013).

36. Kumar, L., Shamsuzzama, Haque, R., Baghel, T. \& Nazir, A. Circular RNAs: the Emerging Class of Non-coding RNAs and Their Potential Role in Human Neurodegenerative Diseases. Mol. Neurobiol. (2016). doi:10.1007/s12035-016-0213-8

37. Liu, S. J. et al. Single-cell analysis of long non-coding RNAs in the developing human neocortex. Genome Biol. 17, 67 (2016).

First single-cell analysis of ncRNAs in human brain.

38. Ayana, R., Singh, S. \& Pati, S. Decoding Crucial LncRNAs Implicated in Neurogenesis and Neurological Disorders. Stem Cells Dev. 26, 541-553 (2017).

39. Ramos, A. D., Attenello, F. J. \& Lim, D. A. Uncovering the roles of long noncoding RNAs in neural development and glioma progression. Neurosci. Lett. 625, 70-79 (2016).

40. Ng, S.-Y., Lin, L., Soh, B. S. \& Stanton, L. W. Long noncoding RNAs in development and 
disease of the central nervous system. Trends Genet. 29, 461-468 (2013).

41. Roberts, T. C., Morris, K. V \& Wood, M. J. A. The role of long non-coding RNAs in neurodevelopment, brain function and neurological disease. Philos. Trans. R. Soc. Lond. B. Biol. Sci. 369, (2014).

42. Davis, G. M., Haas, M. A. \& Pocock, R. MicroRNAs: Not 'Fine-Tuners' but Key Regulators of Neuronal Development and Function. Front. Neurol. 6, (2015).

43. Salta, E. et al. A self-organizing miR-132/Ctbp2 circuit regulates bimodal notch signals and glial progenitor fate choice during spinal cord maturation. Dev. Cell 30, 423-36 (2014).

44. Sun, A. X., Crabtree, G. R. \& Yoo, A. S. MicroRNAs: regulators of neuronal fate. Curr. Opin. Cell Biol. 25, 215-221 (2013).

45. Kuwabara, T., Hsieh, J., Nakashima, K., Taira, K. \& Gage, F. H. A small modulatory dsRNA specifies the fate of adult neural stem cells. Cell 116, 779-793 (2004).

46. Li, J.-S. \& Yao, Z.-X. MicroRNAs: Novel Regulators of Oligodendrocyte Differentiation and Potential Therapeutic Targets in Demyelination-Related Diseases. Mol. Neurobiol. 45, 200-212 (2012).

47. Coolen, M. \& Bally-Cuif, L. MicroRNAs in brain development and physiology. Curr. Opin. Neurobiol. 19, 461-470 (2009).

48. Weiß, K., Antoniou, A. \& Schratt, G. Non-coding mechanisms of local mRNA translation in neuronal dendrites. Eur. J. Cell Biol. 1-5 (2015). doi:10.1016/j.ejcb.2015.05.011

49. Zhao, X. et al. A long noncoding RNA contributes to neuropathic pain by silencing Kcna2 in primary afferent neurons. Nat. Neurosci. 16, 1024-31 (2013).

50. Modarresi, F. et al. Natural Antisense Inhibition Results in Transcriptional DeRepression and Gene Upregulation. Nat Biotechnol. 30, 453-59 (2012).

This paper is one of the first reports on endogenous regulation by NATs in the brain and is the first to coin the term 'antagoNATs' for antisense therapeutic targeting of endogenous NATs.

51. Maag, J. L. V. et al. Dynamic expression of long noncoding RNAs and repeat elements in synaptic plasticity. Front. Neurosci. 9, (2015).

52. Bicker, S., Lackinger, M., Weiß, K. \& Schratt, G. MicroRNA-132, -134, and -138: a microRNA troika rules in neuronal dendrites. Cell. Mol. Life Sci. 71, 3987-4005 (2014).

53. Schratt, G. microRNAs at the synapse. Nat. Rev. Neurosci. 10, 842-849 (2009).

54. Smalheiser, N. R. \& B, P. T. R. S. The RNA-centred view of the synapse : non-coding RNAs and synaptic plasticity The RNA-centred view of the synapse : non-coding RNAs and synaptic plasticity. (2014).

55. Alberini, C. M. \& Kandel, E. R. The Regulation of Transcription in Memory Consolidation. Cold Spring Harb. Perspect. Biol. 7, a021741 (2015).

56. Qureshi, I. A. \& Mehler, M. F. Non-coding RNA networks underlying cognitive disorders across the lifespan. Trends Mol. Med. 17, 337-346 (2011).

57. Meng, L. et al. Towards a therapy for Angelman syndrome by targeting a long noncoding RNA. Nature 518, 409-412 (2014).

This is the first paper to demonstrate the potential of therapeutic targeting of long ncRNAs in neurological disorders.

58. St. Laurent, G. \& Wahlestedt, C. Noncoding RNAs: couplers of analog and digital information in nervous system function? Trends Neurosci. 30, 612-621 (2007).

59. Cooper-Knock, J., Kirby, J., Highley, R. \& Shaw, P. J. The Spectrum of C9orf72-mediated Neurodegeneration and Amyotrophic Lateral Sclerosis. Neurotherapeutics 12, 326339 (2015).

60. DeJesus-Hernandez, M. et al. Expanded GGGGCC hexanucleotide repeat in noncoding region of C9ORF72 causes chromosome 9p-linked FTD and ALS. Neuron 
72, 245-56 (2011).

This is one of the first reports on the causal link between ncRNA expansion repeats and FTD and ALS.

61. Lagier-Tourenne, C. et al. Targeted degradation of sense and antisense C9orf72 RNA foci as therapy for ALS and frontotemporal degeneration. Proc. Natl. Acad. Sci. U. S. A. 110, E4530-9 (2013).

This paper demonstrates the therapeutic potential of using ASOs against coding and noncoding RNA in ALS and FTD.

62. $\mathrm{Zu}, \mathrm{T}$. et al. RAN proteins and RNA foci from antisense transcripts in C9ORF72 ALS and frontotemporal dementia. Proc. Natl. Acad. Sci. 110, E4968-E4977 (2013).

This is one of the first papers on the complexity of the C9ORF72 locus.

63. Taylor, J. P., Brown, R. H. \& Cleveland, D. W. Decoding ALS: from genes to mechanism. Nature 539, 197-206 (2016).

64. Moseley, M. L. et al. Bidirectional expression of CUG and CAG expansion transcripts and intranuclear polyglutamine inclusions in spinocerebellar ataxia type 8. Nat. Genet. 38, 758-769 (2006).

This is the first paper to systematically describe the transcriptional complexity in SCA8.

65. Nemes, J. P., Benzow, K. A., Moseley, M. L., Ranum, L. P. \& Koob, M. D. The SCA8 transcript is an antisense RNA to a brain-specific transcript encoding a novel actinbinding protein (KLHL1). Hum. Mol. Genet. 9, 1543-51 (2000).

66. Pastori, C. et al. Comprehensive analysis of the transcriptional landscape of the human FMR1 gene reveals two new long noncoding RNAs differentially expressed in Fragile $X$ syndrome and Fragile X-associated tremor/ataxia syndrome. Hum. Genet. 133, 59-67 (2014).67. Ladd, P. D. et al. An antisense transcript spanning the CGG repeat region of FMR1 is upregulated in premutation carriers but silenced in full mutation individuals. Hum. Mol. Genet. 16, 3174-3187 (2007).

This is the first report of ncRNAs in FXS.

68. Bassell, G. J. \& Warren, S. T. Fragile X Syndrome: Loss of Local mRNA Regulation Alters Synaptic Development and Function. Neuron 60, 201-214 (2008).

69. Khalil, A. M., Faghihi, M. A., Modarresi, F., Brothers, S. P. \& Wahlestedt, C. A novel RNA transcript with antiapoptotic function is silenced in fragile $X$ syndrome. PLoS One 3, (2008).

70. Peschansky, V. J. et al. The long non-coding RNA FMR4 promotes proliferation of human neural precursor cells and epigenetic regulation of gene expression in trans. Mol. Cell. Neurosci. 74, 49-57 (2016).

71. Bañez-Coronel, M. et al. A pathogenic mechanism in huntington's disease involves small CAG-repeated RNAs with neurotoxic activity. PLoS Genet. 8, (2012).

72. Chung, D. W., Rudnicki, D. D., Yu, L. \& Margolis, R. L. A natural antisense transcript at the Huntington's disease repeat locus regulates HTT expression. Hum. Mol. Genet. 20, 3467-3477 (2011).

This is the first study identifying an HD-related ncRNA.

73. Kordasiewicz, H. B. et al. Sustained Therapeutic Reversal of Huntington's Disease by Transient Repression of Huntingtin Synthesis. Neuron 74, 1031-1044 (2012).

74. Burd, C. E. et al. Expression of linear and novel circular forms of an INK4/ARFassociated non-coding RNA correlates with atherosclerosis risk. PLoS Genet. 6, 1-15 (2010).

75. Popov, N. \& Gil, J. Epigenetic regulation of the INK4B-ARF-INK4a locus: In sickness and in health. Epigenetics 5, 685-690 (2010).

76. Züchner, S. et al. Linkage and association study of late-onset Alzheimer disease families linked to 9p21.3. Ann. Hum. Genet. 72, 725-31 (2008).

77. Emanuele, E. et al. Chromosome 9p21.3 genotype is associated with vascular 
dementia and Alzheimer's disease. Neurobiol. Aging 32, 1231-1235 (2011).

78. Arendt, T., Holzer, M. \& Gärtner, U. Neuronal expression of cycline dependent kinase inhibitors of the INK4 family in Alzheimer's disease. J. Neural Transm. 105, 949-960 (1998).

79. Airavaara, M. et al. Identification of novel GDNF isoforms and cis-antisense GDNFOS gene and their regulation in human middle temporal gyrus of Alzheimer disease. $J$. Biol. Chem. 286, 45093-45102 (2011).

80. Sopher, B. L. et al. CTCF regulates ataxin-7 expression through promotion of a convergently transcribed, antisense noncoding RNA. Neuron 70, 1071-84 (2011).

81. Johnson, R. Long non-coding RNAs in Huntington's disease neurodegeneration. Neurobiol. Dis. 46, 245-254 (2012).

82. Xie, Y., Hayden, M. R. \& Xu, B. BDNF overexpression in the forebrain rescues Huntington's disease phenotypes in YAC128 mice. J. Neurosci. 30, 14708-18 (2010).

83. Chakravarty, D. et al. The oestrogen receptor alpha-regulated IncRNA NEAT1 is a critical modulator of prostate cancer. Nat. Commun. 5, 5383 (2014).

84. Ferrer, I., Goutan, E., Marín, C., Rey, M. J. \& Ribalta, T. Brain-derived neurotrophic factor in Huntington disease. Brain Res. 866, 257-61 (2000).

85. Xu, L., Zhang, Z., Xie, T., Zhang, X. \& Dai, T. Inhibition of BDNF-AS Provides Neuroprotection for Retinal Ganglion Cells against Ischemic Injury. PLoS One 11, e0164941 (2016).

86. Bartel, D. P. MicroRNAs: Genomics, Biogenesis, Mechanism, and Function. Cell 116, 281-297 (2004).

87. Santa-Maria, I. et al. Dysregulation of microRNA-219 promotes neurodegeneration through post-transcriptional regulation of tau. J. Clin. Invest. 125, 681-686 (2015).

88. Lehmann, S. M. et al. An unconventional role for miRNA: let-7 activates Toll-like receptor 7 and causes neurodegeneration. Nat. Neurosci. 15, 827-835 (2012).

89. Roshan, R. et al. Brain-specific knockdown of miR-29 results in neuronal cell death and ataxia in mice. RNA 20, 1287-97 (2014).

90. Salta, E. \& De Strooper, B. microRNA-132: a key noncoding RNA operating in the cellular phase of Alzheimer's disease. FASEB J. 31, 424-433 (2017).

91. Lau, P. et al. Alteration of the microRNA network during the progression of Alzheimer's disease. EMBO Mol. Med. 5, 1613-1634 (2013).

This study provides a systematic and comrehensive analysis of microRNA expression profiles in human $A D$ brain.

92. Salta, E., Sierksma, A., Eynden, E. Vanden \& Strooper, B. De. miR- 132 loss derepresses ITPKB and aggravates amyloid and TAU pathology in Alzheimer 's brain. 114 (2016). doi:10.15252/emmm.201606520

93. Wong, H. K. A. et al. De-repression of FOXO3a death axis by microRNA-132 and -212 causes neuronal apoptosis in Alzheimer's disease. Hum. Mol. Genet. 22, 3077-3092 (2013).

94. Ciarlo, E. et al. An intronic ncRNA-dependent regulation of SORL1 expression affecting $A \beta$ formation is upregulated in post-mortem Alzheimer's disease brain samples. Dis. Model. Mech. 6, 424-33 (2013).

95. Andersen, O. M. et al. Molecular Dissection of the Interaction between Amyloid Precursor Protein and Its Neuronal Trafficking Receptor SorLA/LR11 †. Biochemistry 45, 2618-2628 (2006).

96. Massone, S. et al. 17A, a novel non-coding RNA, regulates GABA B alternative splicing and signaling in response to inflammatory stimuli and in Alzheimer disease. Neurobiol. Dis. 41, 308-317 (2011).

97. Daughters, R. S. et al. RNA gain-of-function in spinocerebellar ataxia type 8. PLoS Genet. 5, (2009). 
98. Faghihi, M. \& Wahlestedt, C. Regulatory roles of natural antisense transcripts. Nat. Rev. Mol. cell Biol. 10, 637-643 (2009).

99. Faghihi, M. A. et al. Expression of a noncoding RNA is elevated in Alzheimer's disease and drives rapid feed-forward regulation of beta-secretase. Nat. Med. 14, 723-730 (2008).

100. Hansen, T. B. et al. miRNA-dependent gene silencing involving Ago2-mediated cleavage of a circular antisense RNA. Embo J 30, 4414-4422 (2011).

101. Zhao, Y., Alexandrov, P., Jaber, V. \& Lukiw, W. Deficiency in the Ubiquitin Conjugating Enzyme UBE2A in Alzheimer's Disease (AD) is Linked to Deficits in a Natural Circular miRNA-7 Sponge (circRNA; ciRS-7). Genes (Basel). 7, 116 (2016).

102. Bosco, P., Spada, R., Caniglia, S., Salluzzo, M. G. \& Salemi, M. Cerebellar degenerationrelated autoantigen 1 (CDR1) gene expression in Alzheimer's disease. Neurol. Sci. 35, 1613-1614 (2014).

103. Morais, V. A. et al. PINK1 loss-of-function mutations affect mitochondrial complex I activity via NdufA10 ubiquinone uncoupling. Science 344, 203-7 (2014).

104. Scheele, C. et al. The human PINK1 locus is regulated in vivo by a non-coding natural antisense RNA during modulation of mitochondrial function. BMC Genomics 8, 74 (2007).

105. Muddashetty, R. S. et al. Poly(A)-binding protein is associated with neuronal BC1 and BC200 ribonucleoprotein particles. J. Mol. Biol. 321, 433-445 (2002).

106. Mus, E., Hof, P. R. \& Tiedge, H. Dendritic BC200 RNA in aging and in Alzheimer's disease. Proc. Natl. Acad. Sci. U. S. A. 104, 10679-10684 (2007).

107. Liu, Y. et al. Association between ubiquitin carboxy-terminal hydrolase-L1 S18Y variant and risk of Parkinson's disease: the impact of ethnicity and onset age. Neurol. Sci. 36, 179-188 (2015).

108. Carrieri, C. et al. Expression analysis of the long non-coding RNA antisense to Uchl1 (AS Uchl1) during dopaminergic cells' differentiation in vitro and in neurochemical models of Parkinson's disease. Front. Cell. Neurosci. 9, 114 (2015).

109. Ambrosi, G. et al. Bioenergetic and proteolytic defects in fibroblasts from patients with sporadic Parkinson's disease. Biochim. Biophys. Acta - Mol. Basis Dis. 1842, 13851394 (2014).

110. Tay, Y., Rinn, J. \& Pandolfi, P. P. The multilayered complexity of ceRNA crosstalk and competition. Nature 505, 344-52 (2014).

111. Guttman, M. \& Rinn, J. L. Modular regulatory principles of large non-coding RNAs. Nature 482, 339-46 (2012).

112. Salmena, L., Poliseno, L., Tay, Y., Kats, L. \& Pandolfi, P. P. A ceRNA Hypothesis: The Rosetta Stone of a Hidden RNA Language? Cell 146, 353-358 (2011).

113. Thomson, D. W. \& Dinger, M. E. Endogenous microRNA sponges: evidence and controversy. Nat. Rev. Genet. 17, 272-283 (2016).

114. Hansen, T. B. et al. Natural RNA circles function as efficient microRNA sponges. Nature 495, 384-8 (2013).

One of the first reports on the role of circular RNAs as microRNA sponges.

115. Zhang, K. et al. The C9orf72 repeat expansion disrupts nucleocytoplasmic transport. Nature 525, 56-61 (2015).

116. Tollervey, J. R. et al. Characterizing the RNA targets and position-dependent splicing regulation by TDP-43. Nat. Neurosci. 14, 452-458 (2011).

117. Adriaens, C. et al. p53 induces formation of NEAT1 IncRNA-containing paraspeckles that modulate replication stress response and chemosensitivity. Nat. Med. 22, 861868 (2016).

118. Yamanaka, Y. et al. Antisense RNA controls LRP1 Sense transcript expression through interaction with a chromatin-associated protein, HMGB2. Cell Rep. 11, 967-76 (2015). 
119. Memczak, S. et al. Circular RNAs are a large class of animal RNAs with regulatory potency. Nature 495, 333-338 (2013).

This is one of the first papers on the role of circular RNAs as microRNA sponges.

120. Shi, Z. et al. The circular RNA ciRS-7 promotes APP and BACE1 degradation in an NFKB-dependent manner. FEBS J. 284, 1096-1109 (2017).

121. Junn, E. et al. Repression of alpha-synuclein expression and toxicity by microRNA-7. Proc. Natl. Acad. Sci. U. S. A. 106, 13052-7 (2009).

122. Tan, J. Y. et al. Cross-talking noncoding RNAs contribute to cell-specific neurodegeneration in SCA7. Nat. Struct. Mol. Biol. 21, 955-61 (2014).

123. Kumar, V. et al. Human Disease-Associated Genetic Variation Impacts Large Intergenic Non-Coding RNA Expression. PLoS Genet. 9, (2013).

124. Shi, J. et al. A 3'-UTR polymorphism in the oxidized LDL receptor 1 gene increases Abeta40 load as cerebral amyloid angiopathy in Alzheimer's disease. Acta Neuropathol. 111, 15-20 (2006).

125. Wang, G. et al. Variation in the miRNA-433 Binding Site of FGF20 Confers Risk for Parkinson Disease by Overexpression of $\alpha$-Synuclein. Am. J. Hum. Genet. 82, 283-289 (2008).

126. Rademakers, R. et al. Common variation in the miR-659 binding-site of GRN is a major risk factor for TDP43-positive frontotemporal dementia. Human Molecular Genetics 17, 3631-3642 (2008).

127. Rollinson, S. et al. No association of PGRN 3'UTR rs5848 in frontotemporal lobar degeneration. Neurobiol. Aging 32, 754-5 (2011).

128. Simón-Sánchez, J. et al. Variation at GRN 3'-UTR rs5848 is not associated with a risk of frontotemporal lobar degeneration in Dutch population. PLoS One 4, e7494 (2009).

129. Hsiung, G.-Y. R., Fok, A., Feldman, H. H., Rademakers, R. \& Mackenzie, I. R. A. rs5848 polymorphism and serum progranulin level. J. Neurol. Sci. 300, 28-32 (2011).

130. Nelson, P. T. et al. Reassessment of Risk Genotypes (GRN , TMEM106B , and ABCC9 Variants) Associated With Hippocampal Sclerosis of Aging Pathology. J. Neuropathol. Exp. Neurol. 74, 75-84 (2015).

131. Zhang, B., Wang, A., Xia, C., Lin, Q. \& Chen, C. A single nucleotide polymorphism in primary-microRNA-146a reduces the expression of mature microRNA-146a in patients with Alzheimer's disease and is associated with the pathogenesis of Alzheimer's disease. Mol. Med. Rep. 12, 4037-42 (2015).

132. Vermunt, M. W. et al. Large-scale identification of coregulated enhancer networks in the adult human brain. Cell Rep. 9, 767-779 (2014).

133. Soldner, F. et al. Parkinson-associated risk variant in distal enhancer of $\alpha$-synuclein modulates target gene expression. Nature 533, 95-99 (2016).

134. Bhartiya, D. \& Scaria, V. Genomic variations in non-coding RNAs: Structure, function and regulation. Genomics 107, 59-68 (2016).

135. Qureshi, I. A. \& Mehler, M. F. Epigenetic mechanisms governing the process of neurodegeneration. Mol. Aspects Med. 34, 875-882 (2013).

136. Chevillet, J. R., Lee, I., Briggs, H. A., He, Y. \& Wang, K. Issues and prospects of microRNA-based biomarkers in blood and other body fluids. Molecules 19, 6080-6105 (2014).

137. Sala Frigerio, C. et al. Reduced expression of hsa-miR-27a-3p in CSF of patients with Alzheimer disease. Neurology 81, 2103-6 (2013).

138. Dong, H. et al. Serum MicroRNA profiles serve as novel biomarkers for the diagnosis of alzheimer's disease. Dis. Markers 2015, (2015).

139. Cheng, L. et al. Prognostic serum miRNA biomarkers associated with Alzheimer's disease shows concordance with neuropsychological and neuroimaging assessment. Mol. Psychiatry 20, 1-9 (2014). 
140. Alvarez-Mora, M. I. et al. MicroRNA expression profiling in blood from fragile Xassociated tremor/ataxia syndrome patients. Genes, Brain Behav. 12, 595-603 (2013).

141. Gandhi, R. et al. Circulating MicroRNAs as biomarkers for disease staging in multiple sclerosis. Ann. Neurol. 73, 729-740 (2013).

142. Grasso, M., Piscopo, P., Confaloni, A. \& Denti, M. A. Circulating miRNAs as biomarkers for neurodegenerative disorders. TL - 19. Molecules 19 VN - r, 6891-6910 (2014).

143. Denk, J. et al. MicroRNA profiling of CSF reveals potential biomarkers to detect Alzheimer's disease. PLoS One 10, 1-18 (2015).

144. Moon, J. et al. Early diagnosis of Alzheimer's disease from elevated olfactory mucosal miR-206 level. Sci. Rep. 6, 20364 (2016).

145. Su, Z. et al. Discovery of a biomarker and lead small molecules to target r(GGGGCC)associated defects in c9FTD/ALS. Neuron 83, 1043-50 (2014).

146. Esteller, M. Non-coding RNAs in human disease. Nat. Rev. Genet. 12, 861-74 (2011).

147. Lau, P., Sala Frigerio, C. \& De Strooper, B. Variance in the identification of microRNAs deregulated in Alzheimer's disease and possible role of lincRNAs in the pathology: The need of larger datasets. Ageing Res. Rev. 17, 43-53 (2014).

148. Wahlestedt, C. Targeting long non-coding RNA to therapeutically upregulate gene expression. Nat. Rev. Drug Discov. 12, 433-46 (2013).

This is one of the first reports on therapeutic targeting of long ncRNAs.

149. DeVos, S. L. \& Miller, T. M. Antisense Oligonucleotides: Treating Neurodegeneration at the Level of RNA. Neurotherapeutics 10, 486-497 (2013).

150. Donnelly, C. J. et al. RNA Toxicity from the ALS/FTD C9ORF72 Expansion Is Mitigated by Antisense Intervention. Neuron 80, 415-428 (2013).

This is one of the first papers to propose RNA targeting as a therapeutic approach in ALS and FTD.

151. Sareen, D. et al. Targeting RNA foci in iPSC-derived motor neurons from ALS patients with a C9ORF72 repeat expansion. Sci. Transl. Med. 5, 208 ra149 (2013).

152. Jiang, J. et al. Gain of Toxicity from ALS/FTD-Linked Repeat Expansions in C9ORF72 is Alleviated by Antisense Oligonucleotides Targeting GGGGCC-Containing RNAs. Neuron 90, 535-550 (2016).

153. Kramer, N. J. et al. Spt4 selectively regulates the expression of C9orf72 sense and antisense mutant transcripts. Science (80-. ). 353, 708-712 (2016).

154. Modarresi, F. et al. Knockdown of BACE1-AS Nonprotein-Coding Transcript Modulates Beta-Amyloid-Related Hippocampal Neurogenesis. Int. J. Alzheimers. Dis. 2011, 929042 (2011).

155. Armakola, M. et al. Inhibition of RNA lariat debranching enzyme suppresses TDP-43 toxicity in ALS disease models. Nat. Genet. 44, 1302-9 (2012).

156. DeVos, S. L. \& Miller, T. Direct Intraventricular Delivery of Drugs to the Rodent Central Nervous System. J. Vis. Exp. (2013). doi:10.3791/50326

157. Schoch, K. M. et al. Increased 4R-Tau Induces Pathological Changes in a Human-Tau Mouse Model. Neuron 90, 941-947 (2016).

158. DeVos, S. L. et al. Tau reduction prevents neuronal loss and reverses pathological tau deposition and seeding in mice with tauopathy. Sci. Transl. Med. 9, eaag0481 (2017).

159. Smith, R. A. et al. Antisense oligonucleotide therapy for neurodegenerative disease. J. Clin. Invest. 116, 2290-6 (2006).

160. Hua, Y. et al. Antisense correction of SMN2 splicing in the CNS rescues necrosis in a type III SMA mouse model. Genes Dev. 24, 1634-1644 (2010).

161. Wahlgren, J. et al. Plasma exosomes can deliver exogenous short interfering RNA to monocytes and lymphocytes. Nucleic Acids Res. 40, e130-e130 (2012).

162. Valadi, H. et al. Exosome-mediated transfer of mRNAs and microRNAs is a novel mechanism of genetic exchange between cells. Nat. Cell Biol. 9, 654-659 (2007). 
163. Munoz, J. L. et al. Delivery of Functional Anti-miR-9 by Mesenchymal Stem Cellderived Exosomes to Glioblastoma Multiforme Cells Conferred Chemosensitivity. Mol. Ther. - Nucleic Acids 2, e126 (2013).

164. Alvarez-Erviti, L. et al. Delivery of siRNA to the mouse brain by systemic injection of targeted exosomes. Nat. Biotechnol. 29, 341-345 (2011).

165. Matsui, M. \& Corey, D. R. Non-coding RNAs as drug targets. Nat Rev Drug Discov (2016). doi:10.1038/nrd.2016.117

166. Miller, T. M. et al. An antisense oligonucleotide against SOD1 delivered intrathecally for patients with SOD1 familial amyotrophic lateral sclerosis: a phase 1, randomised, first-in-man study. Lancet Neurol. 12, 435-442 (2013).

This is the first report on a clinical trial using ASOs in neurodegeneration.

167. Finkel, R. S. et al. Treatment of infantile-onset spinal muscular atrophy with nusinersen: a phase 2, open-label, dose-escalation study. Lancet 388, 3017-3026 (2016).

This paper reports the very recent results from the successful clinical trial using ASOs in infants suffering from SMA.

168. Lardenoije, R. et al. The epigenetics of aging and neurodegeneration. Prog. Neurobiol. 131, 21-64 (2015).

169. Zhang, R., Deng, P., Jacobson, D. \& Li, J. B. Evolutionary analysis reveals regulatory and functional landscape of coding and non-coding RNA editing. PLOS Genet. 13, e1006563 (2017).

170. Guffanti, A., Simchovitz, A. \& Soreq, H. Emerging bioinformatics approaches for analysis of NGS-derived coding and non-coding RNAs in neurodegenerative diseases. Front. Cell. Neurosci. 8, 89 (2014).

171. da Sacco, L., Baldassarre, A. \& Masotti, A. Bioinformatics tools and novel challenges in long non-coding RNAs (IncRNAs) functional analysis. Int. J. Mol. Sci. 13, 97-114 (2012).

172. Stahl, P. L. et al. Visualization and analysis of gene expression in tissue sections by spatial transcriptomics. Science (80-. ). 353, 78-82 (2016).

This is one of the first systematic studies using spatial transcriptomics technology.

173. Leone, S. \& Santoro, R. Challenges in the analysis of long noncoding RNA functionality. FEBS Lett. 590, 2342-2353 (2016).

174. Goyal, A. et al. Challenges of CRISPR/Cas9 applications for long non-coding RNA genes. Nucleic Acids Res. gkw883 (2016). doi:10.1093/nar/gkw883

175. Cabili, M. N. et al. Localization and abundance analysis of human IncRNAs at single-cell and single-molecule resolution. Genome Biol. 16, 20 (2015).

176. Bergmann, J. H. \& Spector, D. L. Long non-coding RNAs: modulators of nuclear structure and function. Curr. Opin. Cell Biol. 26, 10-18 (2014).

177. Brown, C. J. et al. The human XIST gene: analysis of a $17 \mathrm{~kb}$ inactive X-specific RNA that contains conserved repeats and is highly localized within the nucleus. Cell 71, 527-42 (1992).

178. Ip, J. Y. \& Nakagawa, S. Long non-coding RNAs in nuclear bodies. Dev. Growth Differ. 54, 44-54 (2012).

179. Chu, C., Spitale, R. C. \& Chang, H. Y. Technologies to probe functions and mechanisms of long noncoding RNAs. Nat. Struct. Mol. Biol. 22, 29-35 (2015).

180. Bonev, B. \& Cavalli, G. Organization and function of the 3D genome. Nat. Rev. Genet. 17, 661-678 (2016).

181. Engreitz, J. M., Ollikainen, N. \& Guttman, M. Long non-coding RNAs: spatial amplifiers that control nuclear structure and gene expression. Nat. Rev. Mol. Cell Biol. 17, 756770 (2016).

182. Yang, Y., Wen, L. \& Zhu, H. Unveiling the hidden function of long non-coding RNA by identifying its major partner-protein. Cell Biosci. 5, 59 (2015). 
183. Ferrè, F., Colantoni, A. \& Helmer-Citterich, M. Revealing protein-IncRNA interaction. Brief. Bioinform. 17, 106-116 (2016).

184. McHugh, C. A. et al. The Xist IncRNA interacts directly with SHARP to silence transcription through HDAC3. Nature 521, 232-236 (2015).

185. Chu, C. et al. Systematic Discovery of Xist RNA Binding Proteins. Cell 161, 404-416 (2015).

186. Quinn, J. J. et al. Revealing long noncoding RNA architecture and functions using domain-specific chromatin isolation by RNA purification. Nat. Biotechnol. 32, 933-940 (2014).

187. Minajigi, A. et al. A comprehensive Xist interactome reveals cohesin repulsion and an RNA-directed chromosome conformation. Science (80-. ). 349, aab2276-aab2276 (2015).

188. Bartonicek, N., Maag, J. L. V. \& Dinger, M. E. Long noncoding RNAs in cancer: mechanisms of action and technological advancements. Mol. Cancer 15, 43 (2016).

189. Buenrostro, J. D. et al. Quantitative analysis of RNA-protein interactions on a massively parallel array reveals biophysical and evolutionary landscapes. Nat. Biotechnol. 32, 562-568 (2014).

190. Loya, C. M., Lu, C. S., Van Vactor, D. \& Fulga, T. A. Transgenic microRNA inhibition with spatiotemporal specificity in intact organisms. Nat. Methods 6, 897-903 (2009).

191. Meng, L. et al. Small RNA zippers lock miRNA molecules and block miRNA function in mammalian cells. Nat. Commun. 8, 13964 (2017).

192. Bassett, A. R. et al. Understanding functional miRNA-target interactions in vivo by site-specific genome engineering. Nat. Commun. 5, 4640 (2014).

193. Klann, T. S. et al. CRISPR-Cas9 epigenome editing enables high-throughput screening for functional regulatory elements in the human genome. Nat. Biotechnol. (2017). doi:10.1038/nbt.3853

194. Dinger, M. E., Pang, K. C., Mercer, T. R. \& Mattick, J. S. Differentiating protein-coding and noncoding RNA: Challenges and ambiguities. PLoS Comput. Biol. 4, (2008).

195. Filippenkov, I. B., Kalinichenko, E. O., Limborska, S. A. \& Dergunova, L. V. Circular RNAs-one of the enigmas of the brain. Neurogenetics 1-6 (2016). doi:10.1007/s10048016-0490-4

196. Salzman, J., Gawad, C., Wang, P. L., Lacayo, N. \& Brown, P. O. Circular RNAs are the predominant transcript isoform from hundreds of human genes in diverse cell types. PLoS One 7, (2012).

197. Ruiz-Orera, J. et al. Long non-coding RNAs as a source of new peptides. Elife 3, e03523 (2014).

198. van Heesch, S. et al. Extensive localization of long noncoding RNAs to the cytosol and mono- and polyribosomal complexes. Genome Biol. 15, R6 (2014).

199. Niazi, F. \& Valadkhan, S. Computational analysis of functional long noncoding RNAs reveals lack of peptide-coding capacity and parallels with 3' UTRs. Rna 18, 825-843 (2012).

200. Guttman, M., Russell, P., Ingolia, N. T., Weissman, J. S. \& Lander, E. S. Ribosome profiling provides evidence that large noncoding RNAs do not encode proteins. Cell 154, 240-251 (2013).

201. Lauressergues, D. et al. Primary transcripts of microRNAs encode regulatory peptides. Nature 520, 90-3 (2015).

This paper reports the protein-coding potential of microRNA primary transcripts.

202. Gendron, T. F. et al. Antisense transcripts of the expanded C9ORF72 hexanucleotide repeat form nuclear RNA foci and undergo repeat-associated non-ATG translation in c9FTD/ALS. Acta Neuropathol. 126, 829-844 (2013).

This is one of the first reports of antisense RAN translation from the C9ORF72 locus. 
203. Ingolia, N. T., Lareau, L. F. \& Weissman, J. S. Ribosome profiling of mouse embryonic stem cells reveals the complexity and dynamics of mammalian proteomes. Cell 147, 789-802 (2011).

204. Legnini, I. et al. Circ-ZNF609 Is a Circular RNA that Can Be Translated and Functions in Myogenesis. Mol. Cell 66, 22-37.e9 (2017).

205. Pamudurti, N. R. et al. Translation of CircRNAs. Mol. Cell 66, 9-21.e7 (2017).

206. Zhao, Y. et al. NONCODE 2016: An informative and valuable data source of long noncoding RNAs. Nucleic Acids Res. 44, D203-D208 (2016).

207. Qureshi, I. A. \& Mehler, M. F. Emerging roles of non-coding RNAs in brain evolution, development, plasticity and disease. Nat. Rev. Neurosci. 13, 528-41 (2012).

208. ENCODE Project et al. An integrated encyclopedia of DNA elements in the human genome. Nature 489, 57-74 (2012).

209. Rands, C. M., Meader, S., Ponting, C. P. \& Lunter, G. $8.2 \%$ of the Human Genome Is Constrained: Variation in Rates of Turnover across Functional Element Classes in the Human Lineage. PLoS Genet. 10, (2014).

210. Graur, D. et al. On the immortality of television sets: 'Function' in the human genome according to the evolution-free gospel of encode. Genome Biol. Evol. 5, 578-590 (2013).

211. Hon, C.-C. et al. An atlas of human long non-coding RNAs with accurate $5^{\prime}$ ends. Nature 543, 199-204 (2017).

This is the most recent systematic documentation of human long ncRNAs.

212. Taft, R. J., Pheasant, M. \& Mattick, J. S. The relationship between non-protein-coding DNA and eukaryotic complexity. BioEssays 29, 288-299 (2007).

213. Liu, G., Mattick, J. S. \& Taft, R. J. A meta-analysis of the genomic and transcriptomic composition of complex life. Cell Cycle 12, 2061-2072 (2013).

214. Mattick, J. S. RNA regulation: a new genetics? Nat. Rev. Genet. 5, 316-323 (2004).

215. Bentwich, l. et al. Identification of hundreds of conserved and nonconserved human microRNAs. Nat. Genet. 37, 766-770 (2005).

216. Lindblad-Toh, K. et al. A high-resolution map of human evolutionary constraint using 29 mammals. Nature 478, 476-82 (2011).

217. Qureshi, I. a \& Mehler, M. F. An evolving view of epigenetic complexity in the brain. Philos. Trans. R. Soc. Lond. B. Biol. Sci. 369, 1-8 (2014).

218. Prabhakar, S., Noonan, J. P., Pääbo, S. \& Rubin, E. M. Accelerated evolution of conserved noncoding sequences in humans. Sci. (New York, NY) 314, 786 (2006).

219. Hu, H. Y. et al. MicroRNA expression and regulation in human, chimpanzee, and macaque brains. PLoS Genet. 7, 13-15 (2011).

220. Clark, B. S. \& Blackshaw, S. Long non-coding RNA-dependent transcriptional regulation in neuronal development and disease. Front. Genet. 5, 1-19 (2014).

221. Ataman, B. et al. Evolution of Osteocrin as an activity-regulated factor in the primate brain. Nature 539, 242-247 (2016).

222. Kwan, K. Y. et al. Species-dependent posttranscriptional regulation of NOS1 by FMRP in the developing cerebral cortex. Cell 149, 899-911 (2012).

223. Johnson, M., Kawasawa, Y. \& Mason, C. Functional and evolutionary insights into human brain development through global transcriptome analysis. Neuron 62, 494509 (2009).

224. Pollard, K. S. et al. An RNA gene expressed during cortical development evolved rapidly in humans. Nature 443, 167-172 (2006).

This is the first report on a human- and brain-specific ncRNA.

225. Somel, M. et al. MicroRNA-driven developmental remodeling in the brain distinguishes humans from other primates. PLoS Biol. 9, (2011).

226. Somel, M., Liu, X. \& Khaitovich, P. Human brain evolution: transcripts, metabolites and 
their regulators. Nat. Rev. Neurosci. 14, 112-127 (2013).

227. Wood, S. H., Craig, T., Li, Y., Merry, B. \& De Magalhães, J. P. Whole transcriptome sequencing of the aging rat brain reveals dynamic RNA changes in the dark matter of the genome. Age (Omaha). 35, 763-776 (2013).

228. Persengiev, S., Kondova, I., Otting, N., Koeppen, A. H. \& Bontrop, R. E. Genome-wide analysis of miRNA expression reveals a potential role for miR-144 in brain aging and spinocerebellar ataxia pathogenesis. Neurobiol. Aging 32, 2316.e17-2316.e27 (2011).

229. Lipovich, L. et al. Developmental Changes in the Transcriptome of Human Cerebral Cortex Tissue: Long Noncoding RNA Transcripts. Cereb. Cortex 1-9 (2013). doi:10.1093/cercor/bhs414

230. Finch, C. E. \& Austad, S. N. Primate aging in the mammalian scheme: The puzzle of extreme variation in brain aging. Age (Omaha). 34, 1075-1091 (2012).

231. Coulson, E. J., Paliga, K., Beyreuther, K. \& Masters, C. L. What the evolution of the amyloid protein precursor supergene family tells us about its function. Neurochem. Int. 36, 175-184 (2000).

232. Holzer, M., Craxton, M., Jakes, R., Arendt, T. \& Goedert, M. Tau gene (MAPT) sequence variation among primates. Gene 341, 313-322 (2004).

233. Siddiqui, I. J., Pervaiz, N. \& Abbasi, A. A. The Parkinson Disease gene SNCA: Evolutionary and structural insights with pathological implication. Sci. Rep. 6, 24475 (2016).

234. Ruzo, A. et al. Discovery of novel isoforms of Huntingtin reveals a new hominidspecific exon. PLoS One 10, 1-13 (2015).

235. Johnson, R. et al. Human accelerated region 1 noncoding RNA is repressed by REST in Huntington's disease. Physiol. Genomics 41, 269-74 (2010).

236. Espuny-Camacho, l. et al. Hallmarks of Alzheimer's Disease in Stem-Cell-Derived Human Neurons Transplanted into Mouse Brain. Neuron 93, 1066-1081.e8 (2017).

This is the first study using an iPSC-based in vivo humanized chimeric human-mouse AD model to demonstrate the human-specific requirements for neurodegeneration.

237. Hagerman, P. J. \& Hagerman, R. J. Fragile X-associated tremor/ataxia syndrome. Ann. N. Y. Acad. Sci. 1338, 58-70 (2015).

238. Femminella, G. D., Ferrara, N. \& Rengo, G. The emerging role of microRNAs in Alzheimer's disease. Front. Physiol. 6, 1-5 (2015).

239. Lee, Y. et al. miR-19, miR-101 and miR-130 co-regulate ATXN1 levels to potentially modulate SCA1 pathogenesis. Nature neuroscience 11, 1137-1139 (2008).

240. da Silva, F. et al. microRNAs involved in Parkinson's disease: A systematic review. Mol. Med. Rep. (2016). doi:10.3892/mmr.2016.5759

241. Tan, H., Poidevin, M., Li, H., Chen, D. \& Jin, P. MicroRNA-277 modulates the neurodegeneration caused by fragile X premutation rCGG repeats. PLoS Genet. 8, (2012).

242. Zongaro, S. et al. The 3' UTR of FMR1 mRNA is a target of miR-101, miR-129-5p and miR-221: Implications for the molecular pathology of FXTAS at the synapse. Hum. Mol. Genet. 22, 1971-1982 (2013).

243. Gaughwin, P. M. et al. Hsa-miR-34b is a plasma-stable microRNA that is elevated in pre-manifest Huntington's disease. Hum. Mol. Genet. 20, 2225-2237 (2011).

244. Johnson, R. et al. Regulation of neural macroRNAs by the transcriptional repressor REST. Rna 15, 85-96 (2009).

245. Hoss, a G. et al. miR-10b-5p expression in Huntington's disease brain relates to age of onset and the extent of striatal involvement. BMC Med Genomics 8, 10 (2015).

246. Packer, A. N., Xing, Y., Harper, S. Q., Jones, L. \& Davidson, B. L. The bifunctional microRNA miR-9/miR-9* regulates REST and CoREST and is downregulated in Huntington's disease. J. Neurosci. 28, 14341-6 (2008). 
247. Gascon, E. \& Gao, F.-B. The Emerging Roles of MicroRNAs in the Pathogenesis of Frontotemporal Dementia-Amyotrophic Lateral Sclerosis (FTD-ALS) Spectrum Disorders. J. Neurogenet. 28, 30-40 (2014).

\section{Acknowledgements}

E.S. receives funding from the Fonds voor Wetenschappelijk Onderzoek (FWO) and the Alzheimer's Association. B.D.S. was supported by a European Research Council (ERC) grant for his miRNA work, and is supported by the FWO, KU Leuven, VIB, and a Methusalem grant from KU Leuven and the Flemish Government. He is further supported by the Opening the Future campaign of the Leuven Universiteit Fonds (LUF). The authors are grateful to C. Sala Frigerio, E. Leucci, A. Sierksma, R. Guerreiro and J. Bras for reading the manuscript and providing critical feedback.

\section{Competing interests statement}

The authors declare no competing interests. 


\section{Box 1 | Technologies to probe ncRNA functions}

Experimental validation of functional associations between noncoding RNAs (ncRNAs) and tissue homeostasis or disease is an absolute requirement for further progress in the field. Addressing functionality is more difficult for the noncoding part of the genome than it is for protein-coding genes. The functional redundancy, low sequence conservation, nuclear localization and genomic overlap of ncRNAs with other coding or noncoding sequences hamper their functional annotation ${ }^{173,174}$. Nevertheless, a plethora of novel techniques is now available to study ncRNAs. Direct visualization of long ncRNAs in single cells and at single-molecule resolution using RNA-fluorescence in situ hybridization (RNA-FISH) has yielded precise cellular and subcellular expression patterns for these molecules that may point towards distinct functional roles for $n$ nRNAs $^{175,176}$. For instance, the particular localization of a ncRNA close to its own site of transcription inside the nucleus may suggest a role in the transcriptional regulation of a proximal locus ${ }^{175,177}$. In addition high-resolution subcellular visualization of IncRNAs has unveiled a role for NEAT1, MALAT1 and MIAT in the formation of nuclear bodies (speckles and paraspeckles) ${ }^{175,176,178}$. Novel RNA-interactome approaches coupled to high-throughput sequencing allow in vivo identification of functional partners of ncRNAs. ChIRP-seq (chromatin isolation by RNA purification-sequencing), CHART (capture hybridization analysis of RNA targets) and RAP (RNA antisense purification) are employed for IncRNA-chromatin complex purification and identification of ncRNAs involved in topologically associating domains and nuclear organization 176,179-181; ChIRP-MS (ChIRPmass spectrometry) and RAP-MS (RAP-mass spectrometry) are used for assessing RNAprotein interactions ${ }^{182-185}$; and CLASH (cross-linking, ligation and sequencing of hybrids) for RNA-RNA pairing ${ }^{179}$. In addition, several modified versions of each of these technologies such as dChIRP (domain-specific ChIRP) ${ }^{186}$ and iDRiP (identification of direct RNA interacting proteins (iDRiP) ${ }^{187}$ ), are emerging. Probing the secondary structure of ncRNAs using SHAPEseq (RNA-selective 2'-hydroxylacylation and primer extention-sequencing), PARS (parallel analysis of RNA structure) or FragSeq (fragmentation sequencing) ${ }^{188}$ and mapping the tertiary structure of ncRNA-protein complexes by high-throughput technologies such as CLIP-seq (cross-linking and immunoprecipitation-sequencing) ${ }^{182}$, RNA-MaP (RNA-massively parallel array) and RNA-MITOMI (RNA-mechanically induced trapping of molecular interactions) can provide key insights into ncRNA function ${ }^{179,189}$. Assigning functional roles to ncRNAs requires loss-of-function approaches. Many were adapted from the mRNA world. Chemically modified antisense oligonucleotides, such as antagomiRs or other synthetic molecules, such as miRNA sponges ${ }^{190}$ and miRNA zippers ${ }^{191}$ against miRNAs, and siRNAs, antagoNATs and ASOs against IncRNAs ${ }^{174}$, have been successfully used to knockdown ncRNAs and unveil their functional roles in the cell. However, there are several limitations when performing ncRNA loss-of-function studies. siRNA approaches, for instance, turn out to be less effective when targeting nuclear transcripts, which is often the case for IncRNAs ${ }^{173,174}$. Novel site-specific genome engineering based on TALEN (transcription activator-like effector nucleases) and the all-dominating CRISPR/Cas9 (clustered regularly interspaced short palindromic repeats/CRISPR-associated protein-9 nuclease) technologies are used to ablate whole ncRNA loci or their regulatory domains ${ }^{173,174,192,193}$. Selective excision of the full or partial ncRNA sequence is not feasible when the ncRNA intersects other genes or when it is transcribed from bidirectional promoters, which would alter the transcription of neighbouring genes ${ }^{174}$. Moreover, the regulatory domains of IncRNA genes 
remain largely uncharacterized and therefore domain-specific targeting is not always an option. Finally, in several cases, IncRNAs exert their function via the process of transcription per se, and therefore targeting the transcriptional product may not lead to a loss-of-function phenotype ${ }^{173,174}$. Interestingly, a recent study demonstrated that only one-third of the IncRNA loci are amenable to CRISPR applications without affecting additional genes ${ }^{174}$. Therefore, phenotypes are best confirmed using orthogonal approaches, such as siRNA, ASOs and CRISPR. 


\section{BOX 2 | Noncoding transcriptome: from linear to three-dimensional network-based thinking}

The genome does not follow simplistic human-created rules, hence, the dichotomy between 'coding' and 'noncoding' genes is an oversimplification, as we discuss in the main text ${ }^{194}$. In fact, protein-coding and noncoding transcribed domains from intergenic, intronic and intragenic regions and from both positive (sense) and negative (antisense) strands ${ }^{5}$ illustrate the highly interlaced structure of the genome and its transcriptome ${ }^{194}$. Accordingly, RNA transcribed from a single DNA locus has multiple embedded functions and can give rise to several functional products ${ }^{194}$ (FIG. 2). For instance, many protein-coding genes can be transcribed in the antisense orientation (bidirectional transcription) and/or into circular transcripts ${ }^{98,195,196}$. Similarly, IncRNAs often encompass small open-reading frames (ORFs) and are sometimes associated with ribosomes, suggesting that they might be involved in de novo protein synthesis ${ }^{197,198}$, although this possibility remains controversial ${ }^{13,199,200}$. Nevertheless, the ability to encode small peptides has been experimentally validated for certain primary miRNA transcripts in plants ${ }^{201}$, microsatellite repeat expansions ${ }^{202}$, large intergenic ncRNAs (lincRNAs) ${ }^{203}$, natural antisense transcripts(NATs) ${ }^{98}$ and for the more recently identified circular RNAs (circRNAs) ${ }^{196,204,205}$.

Continuous research has yielded an inventory of tens of thousands of ncRNAs ${ }^{206}$. The arbitrary designation of 'small' or 'long' (cut-off set at 200-400 nucleotides) has no functional foundation but rather reflects the technical aspects of biochemical RNA fractionation approaches. ncRNAs are expressed in a highly cell type-, subcellular compartment-, developmental stage- and environmental context-dependent manner ${ }^{41,207}$, and their flexible modular nature allows them to develop RNA-RNA, RNA-DNA and RNA-protein interactions ${ }^{12}$.

However, the question of what percentage of ncRNAs in the genome is really functional remains a matter of both semantic (when is a transcript defined as 'functional'?) and pragmatic (how much of the genome is implicated in evolutionary relevant trait specification, biological function or human-specific pathologies?) debate $e^{208-211}$. 


\section{BOX 3 | ncRNAs as putative evolutionary signals of human brain development, ageing and neurodegeneration}

The degree of organismal complexity correlates better with the proportion of ncRNAs in the genome than with the number of protein-coding genes (even when alternative splicing and posttranslational modifications are considered as effectors of protein diversification $)^{25,212-}$ 214 . This suggests that the evolution of biological complexity in eukaryotes largely stems from the increasing complexity of ncRNA biology ${ }^{214-216}$. ncRNA genes exhibit positive selection, accelerated evolution, species lineage-related expansion and specificity, preferential expression in brain and selective association with neural genes ${ }^{36,217-220}$. An appealing hypothesis is that ncRNAs may be mediators of certain behavioural and cognitive traits of higher organisms ${ }^{207}$, as opposed to CNS proteins, which are (apart from some limited examples of innovations in proteins) almost perfectly conserved across mammalian phyla ${ }^{13,54,221-223}$. Illustrating these principles, the rapidly evolving, brain- and human-specific ncRNA HAR1F (human accelerated region 1F) is transcribed from a genomic region that has been subject to intense positive selection since human divergence from the great apes 224 and along with several Piwi-interacting RNAs (piRNAs), lincRNAs, miRNAs and circRNAs is associated with human-specific brain development and function 195,207,219,225,226.

A genome-wide transcriptomic analysis in rat brain revealed that changes in ncRNA expression patterns are more prominent than changes in mRNA expression patterns during ageing ${ }^{227}$. ncRNAs with important functions in synaptic and other homeostatic processes, such as miRNAs 228, IncRNAs $^{229}, \mathrm{BC}^{200}{ }^{106}$ and circRNAs, are differentially regulated in the ageing human brain. Moreover, the notion that the histopathological outcomes of brain ageing display profound differences across species raises the intriguing possibility that ncRNA-mediated regulation may be implicated in ageing-associated human neurological conditions ${ }^{230}$. The features of progressive mild atrophic brain alterations that mammalian laboratory models display are distinct from clinical neurodegenerative disorders associated with advanced ageing, such as cerebrovascular disease, AD and PD ${ }^{230}$. Notably, no species except humans have shown evidence of major neuronal loss or massive cognitive decline that could compare to clinical grade $A D^{230}$, observations that cannot be attributed to primary structure differences of key protein players. The sequences of amyloid- $\beta$ peptide (the main constituent of the amyloid plaques in $A D)^{231}, M A P T$ (the gene for tau, which precipitates into neurofibrillary tangles in tauopathies and $A D)^{232}$, and the genes encoding $\alpha$-synuclein (which is implicated in PD) ${ }^{233}$ and huntingtin (which is implicated in HD) $)^{234}$ are almost identical in humans and other primates and overall highly conserved among vertebrates. The existence of a human-specific natural antisense transcript (PINK1-AS) that is implicated in PD 104 , human-specific antisense ncRNA regulation of the ataxin-7 (ATXN7) locus in SCA7 ${ }^{80}$, and the human-specific repression of HAR1 by REST along with its downregulation in the striatum of HD patients 235 suggest that the evolutionary non-conserved ncRNAs might have an important contribution to human-specific neurodegenerative disorders. Clearly, more experimental evidence is required to support these observationss; however, this will not be straightforward and classic rodent systems may have to be replaced with more relevant 'humanized' models of neurodegeneration ${ }^{236}$. 
Figure 1 | Abundance of annotated loci in human genome. Protein-coding genes account for approximately one third of all annotated genes, whereas long and small ncRNA genes together provide $40 \%$ of the gene set. Pseudogenes (genes derived from protein encoding loci, which have lost their coding potential, but may still exert regulatory functions) are not discussed here, but are also present at high abundance. Numbers are derived from GENCODE, release 26 (http://www.gencodegenes.org/releases/current.html). 
Figure 2 | A three-dimensional transcriptional 'code' implicated in neurodegeneration. The figure depicts examples of pervasive transcription in loci implicated in neurodegeneration that may induce neurotoxicity via distinct and/or overlapping mechanisms. C9ORF72 gives rise to a sense, non-repeat-containing, protein-coding transcript and two repeat-containing ncRNAs (one in the sense and one in the antisense direction). Six dipeptide repeat proteins (repeat-associated non ATG (RAN) polydipeptides) are translated from the repeat-containing transcripts and accumulate in toxic intracellular foci. Three neurotoxicity-inducing mechanisms have been put forward in C9ORF72-related ALS and FTD 63: C9ORF72 loss-offunction, C9ORF72 repeat expansion-related RNA gain-of-function, and generation of toxic dipeptide repeat proteins. Apart from a loss-of-function of FMRP, the protein encoded by the FMR1 locus, RNA toxic gain-of-function has been also implicated in FXS ${ }^{237}$. The FMR1 locus generates three sense transcripts (one protein-coding and two noncoding transcripts) and two antisense noncoding transcripts. A third example is the sense linear protein-coding and antisense circular ncRNA (ciRS-7, acts as a miR-7 sponge) that are both transcribed from the CDR1 locus. ciRS-7 downregulation (RNA loss-of-function) in human AD brain has been associated with deficits in ubiquitin-mediated amyloid- $\beta$ clearance in $A D{ }^{101,120}$. Angled arrows indicate the direction of transcription of sense and antisense transcripts. ncRNA transcripts can have a feedback regulatory effect on the protein coding mRNAs (dotted lines). The combinatory transcriptional outcome of each locus affects the homeostatic balance in CNS and its disruption can impact neurodegenerative processes. 
Figure 3 I ncRNA mechanisms in neurodegeneration. ncRNAs associated with neurodegenerative disorders may act at multiple functional levels to regulate a spectrum of molecular processes. ncRNAs can act as scaffolds to recruit transcriptional activators or repressors to chromatin-modifying complexes close to gene promoters and epigenetically regulate gene expression in the nucleus. ncRNA regulation can induce preferential inclusion or exclusion of exons thereby shifting mRNA splicing patterns towards certain alternatively spliced isoforms. ncRNAs in both the nucleus and the cytoplasm can bind proteins and other RNA molecules (for example, mRNAs, IncRNAs and miRNAs) to titrate them away from their physiological places of function or guide them to different intracellular compartments. Binding of ncRNAs onto protein-coding transcripts may affect the stability of the latter and either protect them from degradation (mRNA stability) or induce their decay by, for instance, employing the cellular components of RNA interference (RISC). Translational induction or inhibition is another regulatory outcome of ncRNA-mRNA interactions in the cytoplasm. ncRNA, noncoding RNA; DRPs, dipeptide repeat proteins; Pol II, RNA polymerase II; RISC, RNA-induced silencing complex 
Table 1 | ncRNAs involved in neurodegenerative disorders

\begin{tabular}{|c|c|c|c|c|c|c|}
\hline & Epigenetics & RNAi & Splicing & $\begin{array}{c}\text { mRNA } \\
\text { stability }\end{array}$ & Translation & Sequestration \\
\hline AD & ANRIL $^{75}$ & $\operatorname{miRNA}^{238}$ & $\begin{array}{c}\text { SORL1-AS }^{94} \\
\text { 17A }^{96}\end{array}$ & $\begin{array}{c}\text { BACE1-AS }{ }^{99} \\
\text { ciRS-7 } 100\end{array}$ & BC200 105 & LRP1-AS ${ }^{118}$ \\
\hline SCA & SCAANT1 ${ }^{80}$ & $\operatorname{miRNA}^{228,239}$ & ATXNOS ${ }^{97}$ & & & Inc-SCA7 122 \\
\hline PD & ND & $\operatorname{miRNA}^{240}$ & & PINK1-AS ${ }^{104}$ & UCHL1-AS ${ }^{108}$ & ciRS-7 $7^{114}$ \\
\hline $\begin{array}{l}\text { FXS/ } \\
\text { FXTAS }\end{array}$ & & $\operatorname{miRNA}^{241,242}$ & FMR6 $^{66}$ & FMR6 ${ }^{66}$ & & FMR4 ${ }^{67,69}$ \\
\hline HD & $\begin{array}{l}\text { BDNF-AS }^{81} \\
\text { TUG1 }^{81} \\
\text { MEG3 }^{81} \\
\text { NEAT1 }^{81} \\
\text { TUNA }^{81}\end{array}$ & $\begin{array}{c}\text { miRNA }^{243-246} \\
\text { HTT-AS }\end{array}$ & & & & \\
\hline FTD/ALS & & miRNA $^{247}$ & & & & $\begin{array}{c}\text { C9ORF72 }^{63,115} \\
\text { MALAT1 }^{116} \\
\text { NEAT1 }^{116}\end{array}$ \\
\hline
\end{tabular}

$A D$, Alzheimer's disease; SCA, spinocerebellar ataxia; PD, Parkinson's disease; FXS, fragile $X$ syndrome; FXTAS, fragile X-associated tremor/ataxia syndrome; HD, Huntington's disease; FTD, frontotemporal dementia; ALS, amyotrophic lateral sclerosis 
[Au: Please add glossary definitions here in the order in which the terms first appear in the text (main text then display items). I've highlighted some terms in blue or in comments boxes in the text that I feel would benefit from such definitions. Other terms of course could also be defined. Note that the term in the glossary should match the term used in the text exactly and the definition should ideally be no more than a sentence. No references can be cited in glossary definitions.]

Long ncRNAs

Non protein-coding transcripts longer than 200-400 nucleotides including multiple diverse RNA species

\section{Circular RNAs}

Covalently closed, single-stranded transcripts produced by back-splicing of exons in precursor mRNAs

microRNAs

Small (20-25 nucleotides) non protein-coding regulatory RNA molecules involved in posttranscriptional regulation

\section{Endogenous small-interfering RNAs}

Small (21-26 nucleotides) non protein-coding regulatory RNAs produced from endogenous double-stranded RNA precursors and involved in posttranscriptional silencing

Small nucleolar (sno)-derived RNAs

Small (17-30 nucleotides) non protein-coding regulatory RNAs that are derived from the processing of small nucleolar RNAs and are implicated in gene silencing

\section{PIWI-interacting RNAs}

Small (26-33 nuletides) non protein-coding regulatory RNAs involved in epigenetic and posttranscriptional gene silencing via interaction with PPIWI proteins

Long natural antisense transcripts

Long (>200-400 nucleotides) RNA molecules that are transcribed from the opposite DNA strand, they partially overlap with the sense transcript and often regulate its transcription, splicing or stability

\section{Enhancer ncRNAs}

Non protein-coding RNAs that are transcribed from enhancer DNA loci and are implicated in the regulation of gene transcription

\section{Convergent transcription}

The simultaneous transcription from two closely positioned promoters in the sense and antisense orientation with the RNA polymerases heading towards each other

FTLD-TDP

Frontotemporal lobar degeneration with tau-negative, ubiquitin-positive inclusions which contain TAR DNA-binding protein 43 (TDP-43) 


\section{Seed sequence}

Nucleotides 2-7 in the 5'-end of the miRNA sequence that are crucial for recognizing and binding to complementary sites on target mRNA 3'UTRs 


\section{Key points}

- Even though most of the noncoding RNA (ncRNA) species were initially dismissed as products of spurious transcription, a wide spectrum of ncRNA regulatory mechanisms is now emerging.

- ncRNA expression in brain is dynamically regulated in an activity-dependent and spatiotemporally controlled manner suggesting very precise regulatory roles in brain development and function.

- The intricate transcriptional output of genomic loci may impact human brain evolution and explain specific vulnerability to neurodegeneration.

- ncRNA expression and function is perturbed in neurodegenerative disorders and genetic variations in ncRNA networks can be associated to disease risk.

- Understanding the mechanistic aspects of ncRNA function in the central nervous system and how ncRNA dysfunction may lead to neurodegenerative disorders is likely to offer new diagnostic and therapeutic approaches for these diseases.

\section{Author biographies}

Evgenia Salta received her PhD in molecular pharmacology from the Aristotle University of Thessaloniki, Greece, where she studied species transmissibility of prion diseases. She is currently staff scientist in the VIB Center for Brain and Disease Research. Her current research focuses on the role of microRNAs in Alzheimer's disease.

Bart De Strooper is Professor at the KU Leuven and the VIB Center for Brain and Disease Research and incoming director of the UK Dementia Research institute at UCL, London. He is $\mathrm{MD}$ and $\mathrm{PhD}$ and his research interest is Alzheimer's disease. His major findings where the proteases (ADAM10 and gamma-secretases) responsible for Notch signalling and the demonstration that they are responsible for the generation of amyloid- $\beta$ in Alzheimer's Disease. 